\title{
Wireless technologies, medical applications and future challenges in WBAN: a survey
}

\author{
Houssein Taleb $^{1} \cdot$ Abbass Nasser $^{2} \cdot$ Guillaume Andrieux $^{1} \cdot$ Nour Charara $^{2} \cdot$ Eduardo Motta Cruz $^{1}$
}

Accepted: 30 August 2021 / Published online: 21 September 2021

(C) The Author(s), under exclusive licence to Springer Science+Business Media, LLC, part of Springer Nature 2021

\begin{abstract}
Interest and need for Wireless Body Area Networks (WBANs) have significantly increased recently. WBAN consists of miniaturized sensors designed to collect and transmit data through wireless network, enabling medical specialists to monitor patients during their normal daily life and providing real time opinions for medical diagnosis. Many wireless technologies have proved themselves in WBAN applications, while others are still under investigations. The choice of the technology to adopt may depend on the disease to monitor and the performance requirements, i.e. reliability, latency and data rate. In addition, the suitable sensor is essential when seeking to extract the data related to a medical measure. This paper aims at surveying the wireless technologies used in WBAN systems. In addition to a detailed survey on the existing technologies, the use of the emerging Low Power Wide Area Network (LPWAN) technologies, and the future 5G, B5G and $6 \mathrm{G}$ is investigated, where the suitability of these technologies to WBAN applications is studied from several perspectives. Furthermore, medical applications of WBAN are discussed by presenting their methodologies, the adopted wireless technologies and the used sensors. Given that each medical application requires the appropriate sensor to extract the data, we highlight a wide range of the sensors used in the market for medical systems. Recent and future challenges in WBAN systems are given related to the power consumption, the emergence of the Internet of Things (IoT) technologies in WBAN and others.
\end{abstract}

Keywords IoT $\cdot$ LoRaWAN $\cdot$ Medical applications $\cdot$ Sensors $\cdot$ WBAN

Houssein Taleb

houssein.taleb@etu.univ-nantes.fr

Abbass Nasser

abbassnasser@auce.edu.lb

Guillaume Andrieux

guillaume.andrieux@univ-nantes.fr

Nour Charara

nourchrara@auce.edu.lb

Eduardo Motta Cruz

eduardo.mottacruz@univ-nantes.fr

1 CNRS, IETR UMR 6164, Universite de Nantes, F-85000 La Roche sur Yon, France

2 ICCS-Lab, Computer Science Department, American University of Culture and Education (AUCE), Beirut, Lebanon

\section{Introduction}

Our world is facing a rapid growth of population accompanied with an increase in the average lifetime of individuals. According to a study presented by the World Health Organization (WHO), the population beyond 60 years of age would be around 2.1 billions by the year 2050 [1]. According to another WHO study, the rate of deaths in chronic diseases (such as diabetes, Parkinson's, asthma, etc.) is increasing and will reach $66 \%$ in 2030 [2]. One of the reasons of this increasing is the late diagnosis of the patients. Early detection and effective monitoring would mitigate the impact of such diseases and decrease the healthcare cost. Besides the ageing population challenge, the modern lifestyle raises several health issues such as the inappropriate nourishment and the low physical activity leading to several expensive chronic diseases. Recently, the advances in wireless technologies ensure the remote monitoring of the patients. Many technologies have 
emerged the medical systems providing a continuous monitoring of the patients and helping the medical staff to diagnose and thus give medical directions. Being comfortable and not disturbing the daily activity, a network of sensors is installed in or on the patient body to extract some measures related to target diseases or health measures, forming the Wireless Body Area Network (WBAN). Communication between different parts to send data from the patient to the doctor and vice versa is done by several technologies [3, 4]. Due to the various available technologies, choosing the most appropriate technology, among the existing ones, is a challenging task [5]. Each of these technologies has its own functioning characteristics and should respect some constraints when used for healthcare applications. For that, the most common technologies are highlighted in this paper to study their suitability for WBAN medical applications. The WBAN technology usually consists of several small sensors and a centralized hub. According to the disease to monitor and the patient case, the sensors may have different roles, data rate requirement, power consumption and sizes. These sensors sample data and forward it using a wireless technique to the medical center via a power-rechargeable Personal Device (PD) having a greater computing capability relative to the sensors [6]. The continuous monitoring offered by the sensors allows the medical staff to update the patient's condition. Hence, they may let the patients move freely as they are not kept in bed [7]. Moreover, WBAN permits patients to reduce their house or hospital stay thus reducing patients' discomfort and reducing the risk of infection due to the long stay in hospitals $[8,9]$, especially in the case of an infectious pandemic like Covid-19 where the social distancing is needed for preventing the spread of the disease [10]. Also, WBAN may provide real time and secure collection and processing of medical data at the time where the hospitals were no longer able to receive more patients [11]. Thus, WBAN is one of the solutions that can reduces the one-to-one transmission of Covid-19 patients in order to stop the chain of this deadly pandemic [12].

Sensors exist in a very large numbers on the market, in order to record physiological parameters during daily normal activity such as body temperature, heart rate, blood glucose rate and many other uses in several medical systems. Further, the requirements of these sensors, such as the needed data rate and the battery lifetime, may depend on the disease itself. This fact makes the choice of the suitable technology used to transfer the data from the patient body to the medical staff dependent on several parameters such as the reliability, the power consumption, the latency, weight, size, costs, etc. An overall pictorial representation of WBAN is presented in Fig. 1. The figure shows a remote medical monitoring system using WBAN networks, where multiple medical sensors are deployed on the patient's body to measure multiple physiological parameters. The measured data will then be sent to the medical team over a network so that they can monitor the patient's situation.

\subsection{Business impact}

In Europe, in 2017 the healthcare expenditure is about $10 \%$ relative to GDP [13]. In USA, it is estimated that health care spending reached 3.81 trillion dollars in 2019 and would increase to 4.01 trillion dollars in 2020 . It is projected that by 2028 , health care spending in USA would reach 6.19 trillion dollars, where it is estimated that prices for medical services would grow at an average annual rate of $2.4 \%$ from 2019 to 2028 [14].

The insertion of WBAN in the medical applications generates great effects on the industrial and research world. Referring to a study published by Ericson, the revenue for ICT players will reach about 1307 billion dollars in 2026 with a rate of $11 \%$ for healthcare [15]. The spread of WBANs and the wide use of wireless technologies will benefit the economy and may reduce the medical bill.

\subsection{Related works}

Several surveys have detailed WBAN-related technologies and issues. Authors of [16] surveys the latest research of the coexistence of the wireless technologies and the interference mitigation in WBAN. The researchers in [17] discuss the latest developments in wireless technologies available for WBAN systems. The architecture, technologies, challenges and different aspects of WBAN are illustrated in [18], which describes the architectural limitations of the existing WBAN communication framework. In [19], the applications promoted by the WBAN system, fault tolerance, coexistence management and power consumption are discussed. Reference [20] discusses the WBAN communication types and present a comparison among some WBAN medical projects in terms of the used technologies. The work of [21] compares some low-power communication technologies based on the data rate, frequency band, security, topology, range and others, and focuses primarily on remote monitoring of old or chronically ill people. In [22], the paper focuses on the latest LPWAN wireless technologies that can be considered as the next-generation solution for WBAN healthcare monitoring. The main characteristics of WBAN systems with a list of the requirements of possible WBAN applications are also surveyed in [23], where the authors present the key issues of WBAN systems such as the energy consumption minimization problem, the coexistence of the wireless technologies and others. In [24], the authors survey the enabling wireless technologies for WBAN systems, the 
Fig. 1 Overall representation about WBAN

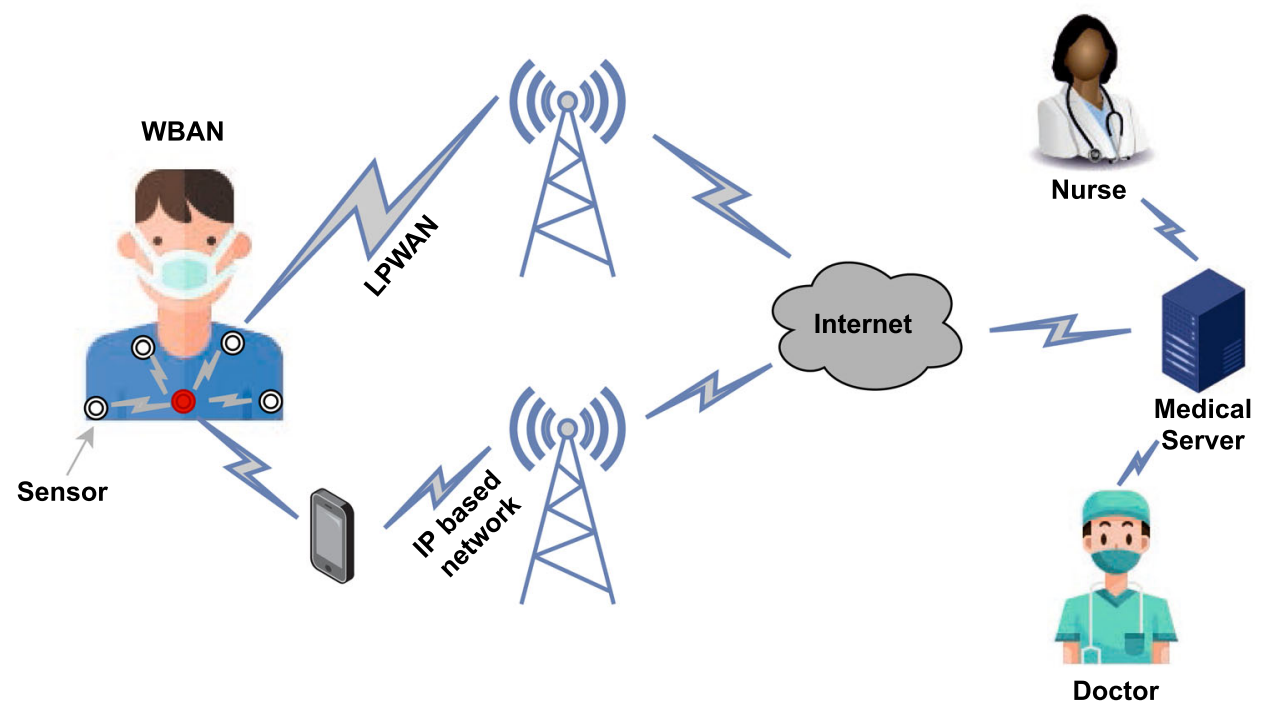

sensor devices, the application scenarios, and finally the interconnection of WBANs. In [25], the work delves into the ways in which the wireless technologies are transforming the IoT healthcare systems and outlines the future path for improving the Quality of Service using the new technologies. The authors of [26] investigate the functions of WBAN, its technologies, and a broader view on WBAN from the perspective of security and authentication. The reference [27] present a survey on the features of WBAN systems, especially communication, sensors, application requirements and security aspects. In [28], several types of miniaturized sensors used in WBAN are presented.

\subsection{Contributions and organisation}

Despite the wide coverage offered by these surveys on the WBAN systems, several points are still not developed, such as the strong and the weak points of the classical and future wireless technologies (WiFi, Bluetooth, ZigBee, B5G, etc) and the IoT technologies (LoRa, Sigfox, etc) when used in medical applications. In addition, to date, there is no work summarizing academic research focused on widely spread diseases (such as diabetes, Parkinson's disease, asthma and others), nor on the characteristics of the sensors used in the market to measure medical parameters for WBAN systems.

Then, the main contributions of this work are as follows:

- A comparison between main wireless technologies is proposed, oriented towards medical applications. We focus on the performance and limitations of each wireless technology in WBAN systems. Besides the traditional wireless technologies, the WBAN perspectives of the $5 \mathrm{G}, \mathrm{B} 5 \mathrm{G}$ and $6 \mathrm{G}$ are presented, and an investigation on the adoption of IoT technologies is proposed, with a focus on the emerging use of LoRa.

- Furthermore, we present a review on the methodologies and the features of some medical systems related to a broad range of diseases investigated in the literature. The technologies and the sensors suitable for each of these diseases are given providing a wide view on the suitability of the technologies to adopt.

- Commercial sensors used to monitor the diseases and found in the market for medical applications are also surveyed.

- Finally, we have detailed the existing challenges and open issues in the domain of healthcare systems.

The rest of this paper is organized as follows. An overview on WBAN systems and their characteristics and features is presented in Sect. 2. Then, a review of the main wireless technologies proposed for WBAN with a comparison between their merits and demerits is detailed in Sect. 3. In Sect. 4, a classification of some WBAN medical systems with their features, methodology and used technologies are presented. We provide some commercially sensors used in WBAN medical systems in Sect. 5. In Sect. 6 , we present the main challenges with a discussion of open research problems. Finally, we conclude our work in the last section.

\section{WBAN overview}

In this section, we present an overview on the architecture and the specifics of WBANs related to power consumption, latency, privacy, criticality of data and interference. 


\subsection{WBAN architecture}

In WBANs, sensors are responsible for detecting physiological data, converting it into digital form and transmitting it to an access point. They can be placed on the human body (on-body sensors), near the body (wearable sensors), or implanted under the skin (in-body sensors). There are two types of sensors: the first type only measures data and sends it [29]. The second type called actuators, measure and send data as the first type and in addition, it can administer medication based on the collected data. The collected data are sent through wireless technologies to the medical server, where it can be analyzed and stored. This can be applied based on 2, 3 or 4 tiers depending on the architecture of the system and depending on the used technologies [20]. The most popular architecture proposed in the literature for WBANs consists of three tiers as shown in figure 2: intra-WBAN, inter-WBAN and beyond-WBAN [20].

\subsubsection{Intra-WBAN}

Intra-WBAN refers first to the communication between the sensors and the PD, second between the sensors themselves [29]. Some existing schemes use cables to connect sensors directly with a PD, thereby avoiding the need for wireless network in the first tier [20]. In other schemes, data can be transmitted wirelessly to a coordinator or master node for further forwarding to the PD (multi-hop WBAN) or can be transmitted directly to the PD (single-hop WBAN) which forwards the data after processing to an Access Point (AP). The multi-hop and single-hop WBAN scenarios are described below:

Multi-hop WBAN: This kind of topology is adopted where the nodes range is short. Thus, to reach the PD, the message sent by a node may pass via the intermediate nodes.

Single-hop WBAN: in single-hop architecture, the PD receives the data directly from the nodes [20]. The power in single-hop configuration is higher than the one of multiHop but the delay is lower.

\subsubsection{Inter-WBAN}

Inter-WBAN includes the communication between the PD and the AP using wireless technologies. Inter-WBAN tier connects WBANs with other networks that can be accessed by Internet. Inter-WBAN communication has two types of architecture: Infrastructure based architecture, which is used in most WBAN applications providing a more secure communication and Ad-hoc based architecture allowing for a quick and flexible disposition [30].

\subsubsection{Beyond-WBAN}

Beyond-WBAN refers to the communication between the AP and the remote medical center. A gateway is used as a bridge to perform the communication via Internet or cellular networks to transmit data to the medical staff. After receiving the data, medical specialists are allowed to analyse it to monitor the patient in order to provide necessary health advice [29]. This tier allows also to restore patient medical information which can be necessary to plan for an appropriate treatment [30].

\subsection{WBAN characteristics}

\subsubsection{Power consumption}

Batteries of small size are used to power the sensors in WBAN systems. Some of these batteries, especially for the

Fig. 2 Three-tiers WBAN architecture

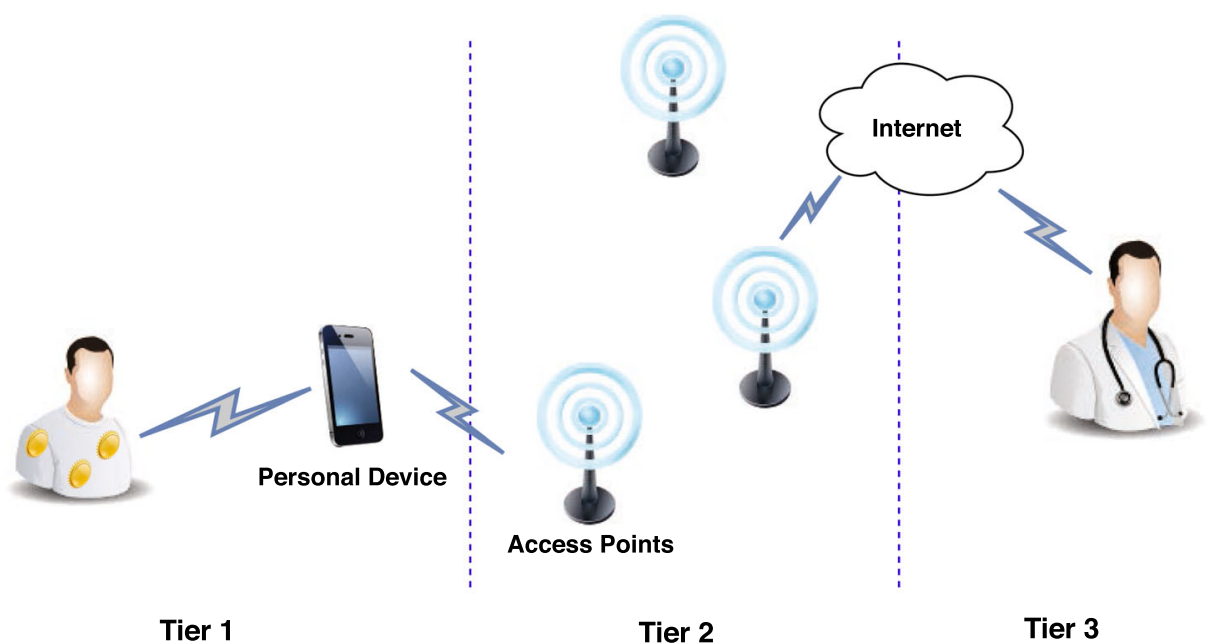


implanted sensors, are irreplaceable or must operate for multiple years without been recharged where replacing them may cause severe pain [29]. Therefore, the goal in WBAN is to obtain an efficient power system while using miniaturized batteries. The first point to consider to obtain an efficient power system is choosing a power efficient wireless technology for WBAN, which is discussed in the next section. Second, we have to let devices to go into sleep mode most of the time, in order to reduce the energy consumption. Third, minimizing the interference, since interference increases the energy consumption of a WBAN system where data should be transmitted more than one time [29].

\subsubsection{Latency}

Latency corresponds to the time taken by the data to travel from a device to another one[22]. It varies from values below one $\mathrm{ms}$ for $5 \mathrm{G} / 6 \mathrm{G}$ to a few tens of milliseconds for $3 \mathrm{G} / 4 \mathrm{G}$, and can exceed one second for LPWAN technologies [31]. The low latency of data transmission ensures the successful transmission of real-time data to the medical center. The WBAN medical applications may be life critical if they cannot tolerate delays, especially in emergency situations, where delayed delivery of data may lead to patient death [22].

\subsubsection{Privacy and security}

Privacy and security of the patient's medical data are two indispensable requirements for WBANs. Privacy means that medical data, which is a highly sensitive form of personal data, must not be disclosed by people who do not have permission to use or view it, so that patients' medical information is not abused. Security in WBANs refers to the protection of a patient's medical data during collection, transmission and storage [22]. Any false data received by the medical staff may cause wrong treatments, and thus may lead to the patient death. As in traditional networks, WBANs have many security requirements [32], the main ones are as follows:

Availability: this requirement aims to ensure that the patient's data can be accessed by the medical staff anytime it is required. Especially for life-saving data which may result in loss of life in case of non-availability of data [30].

Confidentiality: corresponds to the protection of medical data from disclosure. Data eavesdropping breaches privacy which may cause a fatal damage towards the patient. Encryption of medical data over a secure channel provides the confidentiality of data [33].

Authentication: allows to verify that the data is sent by a trusted end device. End devices in WBAN compute a Message Authentication Code (MAC) for the data by sharing a secret key which makes the coordinator realize that the data is received from a trusted end device [34].

Integrity: this requirement aims ensure that the received data has not been manipulated by an adversary using data authentication protocols [33].

Despite the tremendous research work during the last decade, Privacy and Security for WBAN is still a hot topic to study. Many cryptography techniques and algorithms are proposed in several works in the literature to achieve secure WBAN systems. Hereinafter, we list some of the last concerned studies. Authors of [35] propose a strategy of random key deployment scheme. Data in this scheme is transmitted from node to node (through an ad-hoc wireless network) which resists the attack of a cracker. The work of [36] proposes a secure protocol for WBANs where a group of nodes creates trust by forming an authenticated group to associate correctly to the intended patient. In [37], the authors present a physiological-signal-based key agreement (PSKA), which is a secure scheme for communication within a WBAN. PSKA allows neighbouring sensors in a WBAN to agree on a shared cryptographic key in an authenticated manner and without any need of initialization or setup, using the obtained physiological signals. Dynamic password-based authentication scheme is proposed in [38]. The system uses a custom password computation algorithm in order to make the password for each login dynamic and confidential. In [39], the authors propose a lightweight scheme for WBAN authentication based on two secure protocols, the first for authentication and the second for reauthentication in order to protect the sensors anonymity based on the hash function. The proposed authentication cryptosystem for WBANs in [40] is based on an algorithm using a combination of an elliptical curve cryptography with bi-linear pairing schemes. The proposed study in [41] uses firstly the KBS (Key Generation Procedure) keys and Hash algorithm to improve the WBAN's security. Second, KAISC (Key Generation Algorithm for Inter Sensor Communication) is used for inter WBAN communication. The authors in [42] design a secure key agreement protocol and anonymous authentication for WBAN using XOR and simple hash operations. In [43], the work explores the use of biometric characteristics to provide a secure data communication in a WBAN.

\subsubsection{Real time WBAN applications and critical data}

In some medical WBAN applications, the data is critical and cannot tolerate a long response time [44]. Hence, realtime transmission is required with guaranteed performance. Sensors in real-time WBAN applications should instantaneously sense and transmit feedback to the medical staff to process the obtained information while achieving a bounded delay latency. With recent advances of WBAN 
systems, real-time applications have attracted prominent attention from researchers. The work of [45] investigates real time sensors to diagnose the cardiac patients. Also in [46], a WBAN system for electroencephalogram real time monitoring is proposed, where physicians can monitor real time signals via Internet. Another real time WBAN application is proposed in [47] where electrocardiogram measurements are sent to a smartphone in real time.

In real time WBAN applications, sensors may differ in their criticality while transmitting their data [48], where the degree of criticality for each sensor depends on its emergency level and on the nature of the measured data [23]. Distinguishing the criticality of sensors is an important issue to assign a specific priority level to each sensor in WBANs. This distinction between non critical and critical data and, therefore, between high priority and low priority allows data to be scheduled in order to minimize collisions. Moreover, the same sensor may be in different critical states [19]. If the data gathered by a sensor exceeds the value of normal threshold, this indicates that the data has a high critical level and it should be transmitted with high priority. Minimum delay and high reliability level are two prerequisites for data of high criticality, e.g. the data sent by an application for monitoring of elderly heart patients. Moreover, by determining the level of criticality, doctors can examine patients according to their level criticality, which enhances the physician time efficiency [49].

\subsubsection{Interference mitigation}

The ever growing of WBANs results in increasing the interference, which is a real challenge [50]. The interference can affect the performance of networks and may also affect the power consumption of the sensors as a result of the frequent re-transmission when the interference prevents the data to arrive correctly to its destination [50]. As shown in Fig. 3, the interference in WBAN systems can divided into two kinds. First, intra-network interference, as a result of the asynchronous transmissions between near sensors. Second, the inter-network interference, where external sources operating at the same frequency bands of WBAN cause interference [51]. Inter-network interference may also occur when the data of two or more WBANs is sent simultaneously. To ensure the quality of the signal in WBAN, it is necessary to avoid or mitigate these two forms of interference [52]. Authors in [53] and [50] shows that the increase in the number of WBANs and the increase in the data rate have a negative impact on the packet delivery ratio. The effect of distance, orientation of transmitters and receivers, packet size, and the transmission power level on the interference level between the sensors in terms of the packet loss rate was presented in [16] and [54]. In the following, the techniques adopted to mitigate the interference in WBAN systems are presented.

Time Division Multiple Access (TDMA) is a technology that allows a number of nodes to share the same frequency channel by adopting a time slot allocation for one node transmission. TDMA solutions are more suitable for intraWBAN interference rather than inter-WBAN interference mitigation [16]. Moreover, the main problem in TDMA is the delay in critical medical data delivery that may exist when the time frame is divided into time slots less than the number of nodes [55].

In Frequency Division Multiple Access (FDMA), each channel can carry digital data at the same time without interfering with other channels. The limited number of available channels is the main problem in FDMA [16].

Code Division Multiple Access (CDMA) is a multiple access technology, where the users are provided with
Fig. 3 Inter and Intra WBAN interference

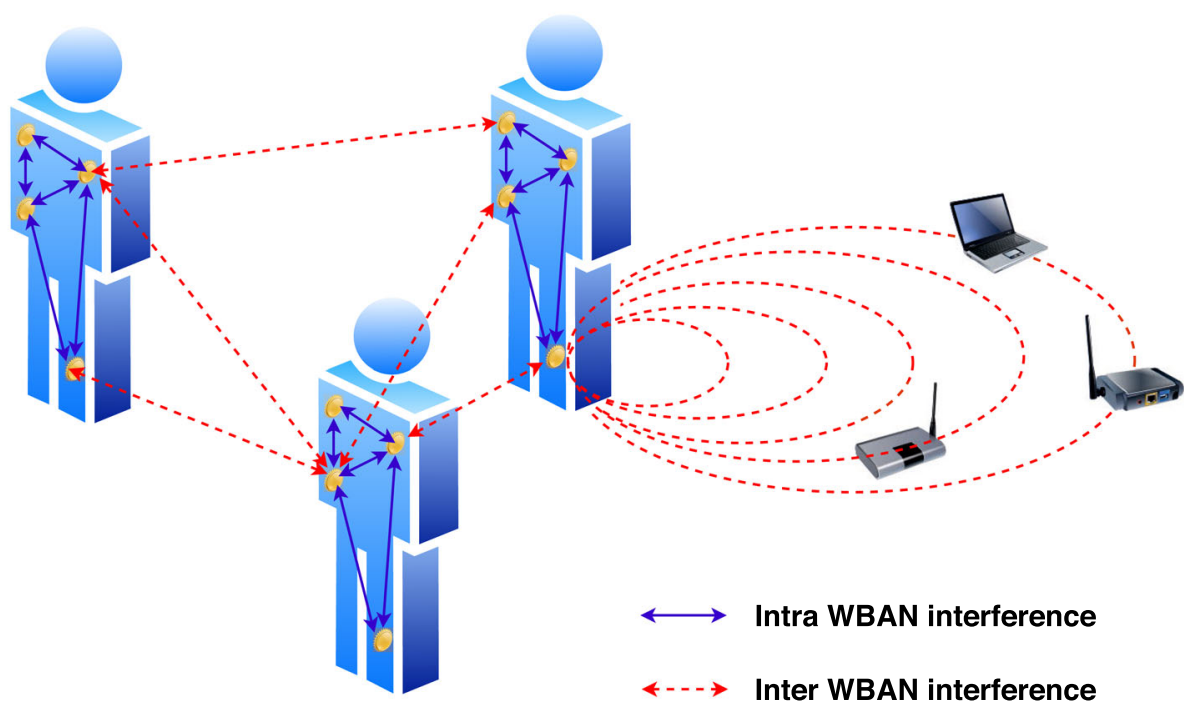


codes, which are orthogonal to each other. This means that all users can use the same frequency and transmit at the same time [56]. CDMA is very effective for multi-sensors transmission scenario in WBANs.

With the emerging of new wireless technologies such as 5G and B5G, several multiple access techniques such as Non-Orthogonal Multiple Access (NOMA) and Sparse Code Multiple Access (SCMA) seem to be promising to alleviate the interference issue [57]. NOMA allows allocating one frequency channel to multiple users at the same time within the same cell, offering a high throughput and low latency [57]. SCMA allows non-orthogonal transmissions of multiple users on the same resource blocks with different codebooks. With sparse codebooks, SCMA reduces the collision between users[58].

Choosing the priority of a sensor can also mitigate the interference. The data collected by each sensor in a WBAN differs in their delay requirements, for example the emergency medical data require minimum delay, so it has the higher privilege to access the channel. Beside the priority of the sensor, it is necessary to classify the WBAN priority among inter-WBAN in multi-WBANs coexistence scenario [59]. To select the critical data among multiple sensors in multi WBANs coexistence scenario, researchers define the health critical index [52] where it considers the sensor priority and the WBAN priority. In [60] an algorithm is proposed where the sensors in multi-WBANs coexistence scenario are sorted from large to small value of the health critical index to select the more critical sensor.

\section{Wireless technologies candidates}

The use of wireless technologies for the exchange of information collected by the sensors, eliminates the needs of cables between the sensor and the access point. The choice of the appropriate wireless technology is an important part of the WBAN systems where an inappropriate technology can result in waste of time and energy. In this section we present the characteristics of the main proposed technologies for WBAN.

\subsection{IEEE.802 standards}

\subsubsection{Bluetooth}

Bluetooth was designed for short range wireless communication, where a number of Bluetooth devices form a short-range personal network known as a piconet [44].A single piconet includes a device which acts as a master and controls up to seven devices known as slaves. A slave device can only communicate with a master device, but cannot directly communicate with other slave devices.
Bluetooth devices operate in the $2.4 \mathrm{GHz}$ ISM band with a coverage range from 1 to $100 \mathrm{~m}$. The maximum data rate is $3 \mathrm{Mbps}$ and the time needed for connection setup and data transfer is about $100 \mathrm{~ms}$ [44]. For WBAN systems, Bluetooth can be usually used for the communication between the sensors and the PD in the first tier, it is free and supported by most devices. It can be used for sensors of relatively high data rate requirement, but it is not an ideal choice in terms of power consumption.

\subsubsection{Bluetooth low energy}

Bluetooth Low Energy (BLE) was designed to wirelessly connect small and low-power devices to mobile terminals. BLE network topology is a star. BLE device can be a part of multiple piconets and can operate both as a master or slave device. Thus, these devices can play multiple roles at the same time which enables the communication between piconets 61,62$]$. BLE offers a data rate up to $1 \mathrm{Mbps}$ [24, which makes it a suitable choice for WBAN applications. Moreover, thanks to the low duty cycle, BLE consumes $90 \%$ lesser than the power required by Bluetooth. This leads to extend the lifetime of the sensor's battery in WBAN systems. But BLE has a range of $10 \mathrm{~m}$, which make it difficult to be used in other than the first tier in WBAN. Synchronization can be done in a few milliseconds compared to Bluetooth seconds which is very valuable for latency-critical devices used in WBAN health-monitoring applications. It uses $2.4 \mathrm{GHz}$ frequency and the time needed for connection setup and data transfer is less than $3 \mathrm{~ms}$ $[21,44]$.

\subsubsection{ZigBee-IEEE 802.15.4}

ZigBee is a wireless technology providing a long battery lifetime, a low data rate and a secure networking as explained below [63]. ZigBee is an easy network to install and configure, it supports various network topologies and a large number of nodes. Its equipment can operate for a few years before the battery needs to be replaced which make it one of the technologies used in many medical applications in WBAN. ZigBee is a secure networking where it offers three levels of security mode to prevent the data from being changed or accessed by attackers [64]. With a range of 100 m, ZigBee operates at $2.4 \mathrm{GHz}, 915 \mathrm{MHz}$ and $868 \mathrm{MHz}$ frequency bands with a data rate of $250 \mathrm{Kbps}, 40 \mathrm{Kbps}$ and $20 \mathrm{Kbps}$ for each of the operating frequencies respectively. For WBAN, ZigBee targets medical applications that require frequent measurements and text-based data transmission with low power consumption. It can be used for the body temperature monitoring, for the pulse monitoring and others [64]. Due to its low data rate, ZigBee is insignificant for some WBAN medical applications which requires high 
data rate and it is difficult to implement it in hospitals for multiple patients [65]. Thus, another significant disadvantage of ZigBee for WBAN applications is due to interference in $2.4 \mathrm{GHz}$ band where many wireless systems operate.

\subsubsection{WiFi-IEEE 802.11}

WiFi provides reliable, secure and fast communication. It is integrated in all smartphones, tablets and laptops. WiFi comes generally with four standards $(802.11 \mathrm{a} / \mathrm{b} / \mathrm{g} / \mathrm{n})$, it can operate in the 2.4 and $5 \mathrm{GHz}$ bands and has a coverage of $100 \mathrm{~m}$ [66]. It is able to deliver data rates of up to 600 Mbps [44]. It is ideally suitable for large amount of data transfers with high-speed wireless connectivity that allows voice calls and video streaming. In WBAN systems, the use of WiFi is not preferred in the first tier due to its high power consumption [65], it is used for the communication between the PD and the APs in the second tier in some WBAN medical applications. As Bluetooth and ZigBee, WiFi occupy the ISM bands, where serious co-channel interference may occur.

\subsubsection{Li-Fi}

The wide usage of the wireless spectrum has many drawbacks such as the interference and the absorption of radio frequency radiations [67]. One of the revolutionary solutions to overcome these issues is Light Fidelity (Li-Fi) for indoor usage and for high speed data network [68]. Li-Fi networks support the transmission of data through illumination of Light Emitting Diode (LED) bulb. It provides a high data rate up to $1 \mathrm{Gbps}$ or even more with a low cost and low power consumption thanks to the use of LED illumination. From the medical perspectives, LED may be available in the homes and hospitals, which makes the LiFi applicable for WBAN medical applications. Furthermore, Li-Fi provides high secure and private connection, low interference and high bandwidths [69]. This helps to protect the privacy of the patient while ensuring a reliable communication. By contrast, the limitation of the use of Li$\mathrm{Fi}$ is due the need to light source. This drawback prevents the cross-walls communications and imposed the communication to be short range [69].

\subsection{LPWAN technologies}

Low Power Wide Area Networks (LPWAN) technologies become very popular in industrial and research communities thanks to their long range and energy-efficient communication. Due to efficient adopted protocols, the lifetime of the end-nodes of the LPWAN-based technologies is of order of years instead of days or months [70]. Hereinafter, we show the main characteristics of five popular LPWAN technologies: LoRa, SigFox, INGENU RPMA, NB-IoT and WiFi HaLow.

\subsubsection{LoRa}

The LoRa (Long Range) Alliance proprietary LPWAN technology consists of two main components, LoRa and LoRaWAN protocol. LoRa is a physical layer modulation scheme used in LPWAN technologies that drawn several research for healthcare systems due to its low cost, long coverage area, and long lifetime of the sensor [71]. LoRa modulation uses orthogonal Spreading Factors (SF) [72]. This enables multiple packets of different SF to be in the same channel concurrently without interference [71]. The number of data bits modulated depends on the used SF, higher SF implies that there are fewer data to encode per second, compared to lower SF. Sending the same amount of data with higher SF needs more transmission time.

In turn, LoRaWAN protocol is a wireless communication protocol developed by LoRa Alliance. The architecture of a LoRaWAN network supports a two-tier topology (see Fig. 4), and it is based on three different types of devices: end-devices, gateway and network server. Such architecture is helpful for WBAN because end-devices collect information and sends them to gateways directly by combining the two tiers 1 and 2 and subsequently eliminating the use of a PD. Then, the data is transmitted from the gateways to the network servers using any types of IP infrastructure such as $4 \mathrm{G}$ or $\mathrm{WiFi}$ as shown in Fig. 4 [73].

LoRaWAN utilizes different Classes A, B and C to serve different applications [74]. End-devices can switch between classes according to the requirements of the application. Class A end-devices initiate an uplink transmission by transmitting the data to the gateway. The time

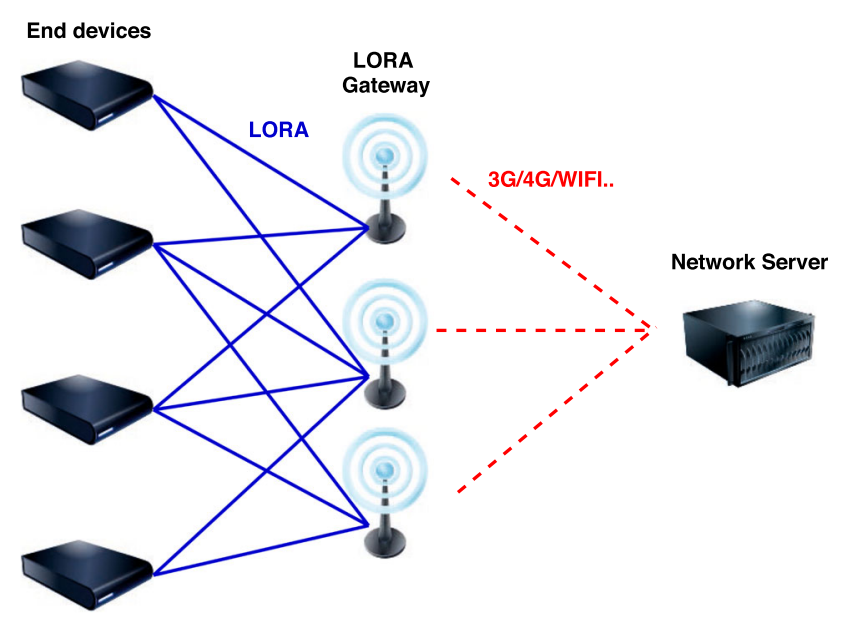

Fig. 4 LoRa architecture 
interval between two consecutive uplink transmissions is scheduled by the end device based on its requirements. After the transmission, the end-device opens two receive windows (RX1 and RX2) to receive an acknowledgment or any other downlink traffic from the gateway as shown in Fig. 5. After the receive windows, the class A device goes to sleep to conserve energy. The gateway has no control on the end device, and any downlink communication requires waiting or the next scheduled uplink transmission. In turn, class B ensures a synchronization between the end device and the gateway when the former functions as actuator. The gateway initiates a downlink transmission by sending a beacon. When receiving the beacon, the end-device initiates the transmissions and open its receive window at the scheduled time as shown in Fig. 5 similarly to Class A. Finally, Class $\mathrm{C}$ end-devices are grid-powered and are always listening to the channel and have almost continuously open receive windows. End devices can receive downlink messages any time whenever it is not in a transmitting state without having to wait for a receive window to open. As a conclusion Class A is the less power, Class $C$ provides end devices with the lowest latency while Class B represents a moderate solution in terms of latency and consumed power. Table 1 summarizes the characteristics of LoRaWAN classes. When projecting LoRaWAN classes to WBAN application, these classes may have different applications and are suitable or not suitable accordingly. Taking into consideration the low power consumption where the system can last up to 5-10 years and the high coverage, where a single gateway can cover an entire city (the coverage is approximately $20 \mathrm{~km}$ in rural regions and reduces to about $2 \mathrm{~km}$ in urban regions), LoRaWAN-based WBAN reduces the cost and limits the complexity of the network enabling to utilize a star network. For instance, the monitoring of patients at hospitals or in their homes can be done by just one base station covering a whole city. Wherefore, LoRa becomes one of the promising technologies for the medical applications in WBAN. Class-A is suitable for the non-continuously

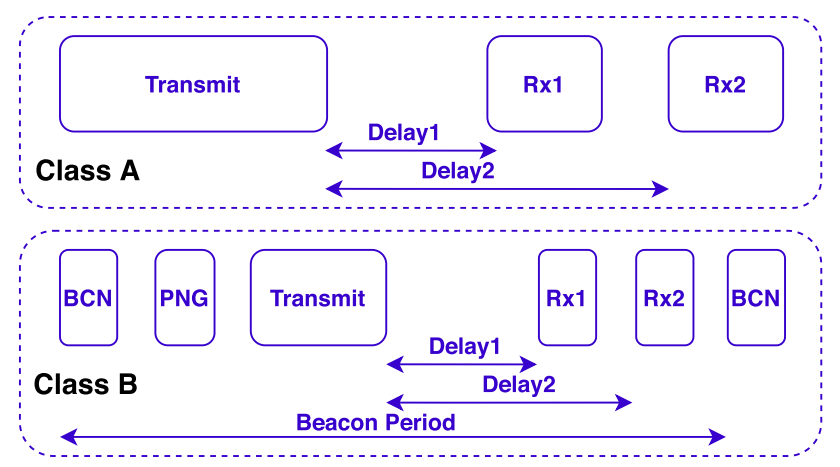

Fig. 5 LoRaWAN device classes A and B monitoring application that does not require a high data rate such as the body temperature monitoring [75] due to the strict regulations of duty cycle (no more than $1 \%$ duty cycle) and due to the low data rate that, can reaches only 50 Kbps [71, 73]. For the continuous applications, when the sensors function as actuator, Class-B can be applied but at the conditions of respecting the duty cycle and admitting some latency due to the synchronization process. By contrast, for other applications such as heart rate monitoring and asthma monitoring, LoRaWAN becomes an unsuitable choice because of the need of a data rate of hundreds of kbps which cannot be provided by LoRa technology. For Class-C, its application becomes limited due to its need to be grid-powered.

\subsubsection{SigFox}

SigFox is a wireless communication network in the category of the LPWAN technology that supports a two-tier topology [76]. Further, the first releases of SigFox technology supported only uplink communication. Later it was evolved into a bidirectional technology [77]. The downlink communication can only precede uplink communication after which the end device should wait to listen for a response from the base station [77]. SigFox was designed to achieve a long-range communication (the coverage is approximately $50 \mathrm{~km}$ in rural regions and reduces to about $10 \mathrm{~km}$ in urban regions) with a low cost, easy connectivity, low power consumption, and low electromagnetic radiation on patients' bodies [77]. By contrast, the application of Sigfox in WBAN systems is considered limited due to several constraints [6]. In fact, SigFox offers only $100 \mathrm{bps}$ data rate, which is insignificant for many medical applications such that asthma monitoring, the monitoring of diabetes patients and many others. Furthermore, there is an issue of reliability due to the use of low received power so the presence of any nearby device can jam the SigFox network. Moreover, SigFox does not provide any valuable collision avoidance mechanism. Therefore, despite its low power consumption and its long range, it remains to do some modification in the SigFox protocol to support the WBAN medical applications.

\subsubsection{Ingenu RPMA}

Ingenu uses a physical multiple access scheme named as random phase multiple access (RPMA) based on the direct sequence spread spectrum technique [78]. It is a technology designed to provide the highest network capacity comparing to LoRa and SigFox where each access point can handle hundreds of thousands of endpoints which would be advantageous in WBAN [78]. Ingenu's range is short compared to LoRa and SigFox, only reaching almost 4.6 
Table 1 LoRa classes

\begin{tabular}{|c|c|c|}
\hline Class-A & Class-B & Class-C \\
\hline Nodes only wake up to send data & Open extra receive window at scheduled times & Always listening to the channel \\
\hline $\begin{array}{l}\text { Battery powered, Most power } \\
\text { efficient }\end{array}$ & $\begin{array}{l}\text { Battery powered, a moderate solution in terms of consumed } \\
\text { power }\end{array}$ & Mains powered, high power consumption \\
\hline $\begin{array}{l}\text { End device initiate the } \\
\text { transmission }\end{array}$ & Gateway can initiate transmission at fixed intervals & $\begin{array}{l}\text { Gateway can initiate the transmission at any } \\
\text { time }\end{array}$ \\
\hline The highest latency & Low latency & No latency \\
\hline
\end{tabular}

$\mathrm{km}$ in urban areas [79]. Also, Ingenu has a robustness to treat the Doppler effect which makes it suitable to work with sensor in mobility scenarios in WBAN [80]. In contrast with most LPWAN technologies, Ingenu operates in the $2.4 \mathrm{GHz}$ ISM band, therefore interference is more probable since $2.4 \mathrm{GHz}$ ISM band is widely used by many other technologies, including WiFi and Bluetooth which can affect the effectivity of RPMA for the medical applications in WBAN in terms of reliability and energy efficiency [78]. Also, Ingenu allows for higher transmission power and antenna gain to be used, but this uses extra power which is not ideal for a WBAN systems [79].

\subsubsection{NB-IoT}

NB-IoT is a narrow-band technology developed for long battery life (more than 10 years), low cost, high capacity (up to 100000 devices per cell), high coverage (approximately $10 \mathrm{~km}$ in rural regions and reduces to about $1 \mathrm{~km}$ in urban regions), data rate up to $230 \mathrm{kbps}$ for the downlink and $250 \mathrm{kbps}$ for the uplink and low bandwidth requirements (needs only $200 \mathrm{kHz}$ ) [81, 82]. For power consumption, [83] indicates that the used NB-IoT chipset consumes over $200 \mathrm{~mW}$ on average for up keeping the network synchronization and almost 350 to $700 \mathrm{~mW}$ during transmission. However, the latency for NB-IoT technology is less than 10 secs, so it cannot be used for emergent and for continuous monitoring applications in WBAN, the high latency makes it more applicable to the delay-tolerant applications. NB-IoT uses single-carrier Frequency Division Multiple Access (FDMA) in uplink and Orthogonal FDMA (OFDMA) in downlink. It achieves excellent coexistence performance with GSM and LTE (long-term evolution) under licensed frequency bands [84]. Also, there is an issue of reliability since only half the messages are acknowledged in NB-IoT due to limited downlink capacity, which is not convenient for WBAN medical applications [85].

\subsubsection{WiFi HaLow}

A low-power and long-range new version of the IEEE 802.11 WiFi standard is called HaLow and is expected to play an important part in IoT and could be useful for connections among smaller and low powered devices in WBAN systems. Unlike the classical WiFi, WiFi HaLow operates at the unlicensed $900 \mathrm{MHz}$ band, offering longer range than classical WiFi (approximately $1 \mathrm{~km}$ ), a high capacity (Up to 8000 devices connected to one AP), a great ability to penetrate walls, a data rate up to $300 \mathrm{Mbps}$ and a lower power connectivity necessary for wearable devices in WBANs (the battery life is up to 10 years). In order to reduce the power consumption, devices using HaLow wake up at defined interval of time [86, 87, 88].

There is a lot of different parameters and characteristics that each technology may offer to the WBAN medical systems. These characteristics may include the communication range, the power consumption, the data rate, the frequency band, the latency and others presented in table 2 . Table 2 summarizes the main characteristics of ZigBee, Bluetooth, BLE, WiFi, SigFox, Lora, Ingenu, NB-IoT and WiFi HaLow where the preference of one technology over another can be made based on the factors indicated above.

The diagram in Fig. 6 summarizes the merits and demerits of Bluetooth, BLE, ZigBee, WiFi, SigFox, Lora, Ingenu, NB-IoT and WiFi HaLow technologies according to a series of criteria including energy consumption, data rate, range, cost $[89,82]$, latency and the maximum number of devices that can be connected at once [90].

As a conclusion, we can say that the choice of a technology depends on the requirement of the sensors, and the requirements of the system and its capability to provide energy. For example, for sensors of very high data rate requirement we can use $\mathrm{WiFi}$. For sensors that need to transmit continuously, LoRa becomes an unsuitable choice but it is an ideal choice to monitor patients that circulates over a large area and uses sensors of low data rate that requires low power consumption. The table and the diagram presented below help us choose the suitable technology for any medical system according to its needs and requirements.

\subsection{Future cellular technologies}

With the ever growing of the wireless communicationbased applications, new cellular technologies have been 
Table 2 The main characteristics of ZigBee, Bluetooth, BLE, WiFi, Sigfox, LoRa, Ingenu, NB-IoT and WiFi HaLow

\begin{tabular}{|c|c|c|c|c|c|c|c|c|c|}
\hline Characteristic & Bluetooth & BLE & ZigBee & $\mathrm{WiFi}$ & LoRaWAN & SigFox & Ingenu & NB-IoT & $\begin{array}{l}\text { WiFi } \\
\text { HaLow }\end{array}$ \\
\hline Operating Frequency & $\begin{array}{l}2400 \\
\mathrm{MHz}\end{array}$ & $\begin{array}{l}2400 \\
\mathrm{MHz}\end{array}$ & $\begin{array}{l}2400,868,915 \\
\text { MHz }\end{array}$ & $\begin{array}{l}2400 \\
\mathrm{MHz} \\
5000 \\
\mathrm{MHz}\end{array}$ & $\begin{array}{l}433 \mathrm{MHz}, \\
868 \\
\mathrm{MHz} \\
915 \mathrm{MHz}\end{array}$ & $\begin{array}{l}915 \\
\mathrm{MHz} \\
868 \\
\mathrm{MHz}\end{array}$ & $\begin{array}{l}2400 \\
\mathrm{MHz}\end{array}$ & $\begin{array}{l}\text { licensed } \\
\text { bands } \\
\text { (e.g., } \\
700,800, \\
\text { and } 900 \\
\text { MHz) }\end{array}$ & $900 \mathrm{MHz}$ \\
\hline Data Rate & $3 \mathrm{Mbps}$ & $1 \mathrm{Mbps}$ & $\begin{array}{l}20 \mathrm{Kbps}(868 \\
\mathrm{MHz}), 40 \\
\mathrm{Kbps}(915 \\
\mathrm{MHz}), 250 \\
\mathrm{Kbps}(2400 \\
\mathrm{MHz})\end{array}$ & $\begin{array}{l}600 \\
\text { Mbps }\end{array}$ & $50 \mathrm{kbps}$ & $100 \mathrm{bps}$ & $\begin{array}{l}230-250 \\
\text { kbps }\end{array}$ & $200 \mathrm{kbps}$ & $\begin{array}{l}\text { up to } 300 \\
\text { Mbps }\end{array}$ \\
\hline Range & $\begin{array}{c}10-100 \\
\mathrm{~m}\end{array}$ & 10 Meters & 100 Meters & $\begin{array}{l}100 \\
\text { Meters }\end{array}$ & $2-20 \mathrm{~km}$ & $\begin{array}{c}10-50 \\
\mathrm{~km}\end{array}$ & $\begin{array}{l}4.6 \mathrm{~km} \text { in } \\
\text { urban } \\
\text { areas }\end{array}$ & $1-10 \mathrm{~km}$ & $\begin{array}{l}\text { Up to } 1 \\
\mathrm{~km}\end{array}$ \\
\hline Network Topology & $\begin{array}{l}\text { Ad-hoc, } \\
\text { star, } \\
\text { mesh }\end{array}$ & $\begin{array}{l}\text { Ad-hoc } \\
\text { piconets }\end{array}$ & Ad-hoc piconets & star & star, mesh & star & star, tree & star & star \\
\hline $\begin{array}{l}\text { Power } \\
\text { Consumption/battery } \\
\text { life }\end{array}$ & $100 \mathrm{~mW}$ & $10 \mathrm{~mW}$ & $30 \mathrm{~mW}$ & $\begin{array}{l}\text { The } \\
\text { higher }\end{array}$ & $\begin{array}{l}\text { Up to } 10 \\
\text { years }\end{array}$ & $\begin{array}{l}10 \mu \mathrm{W}- \\
100 \\
\mathrm{~mW} / \\
\mathrm{Up} \text { to } \\
10 \\
\text { years }\end{array}$ & $\begin{array}{l}\text { Up to } 15 \\
\text { years }\end{array}$ & $\begin{array}{l}200 \mathrm{~mW}- \\
700 \mathrm{~mW} / \\
\text { Up to } 10 \\
\text { years }\end{array}$ & $\begin{array}{l}\text { up to } 10 \\
\text { years }\end{array}$ \\
\hline Bandwidth & $1 \mathrm{MHz}$ & $2 \mathrm{MHz}$ & $2 \mathrm{MHz}$ & $\begin{array}{l}20 \mathrm{MHz}, \\
40 \\
\mathrm{MHz} \\
80 \\
\mathrm{MHz} \\
160 \\
\mathrm{MHz}\end{array}$ & $\begin{array}{l}125 \mathrm{KHz} \\
250 \mathrm{KHz} \\
\text { and } \\
500 \mathrm{KHz}\end{array}$ & $100 \mathrm{~Hz}$ & $1 \mathrm{MHz}$ & $200 \mathrm{kHz}$ & $\begin{array}{c}1,2,4,8,16 \\
\mathrm{MHz}\end{array}$ \\
\hline $\begin{array}{l}\text { Spread Spectrum } \\
\text { Technology }\end{array}$ & FHSS & FHSS & DSSS & DSSS & CSS & BPSK & BPSK & QPSK & $\begin{array}{l}\text { DPSK and } \\
\text { QPSK }\end{array}$ \\
\hline Latency & $\begin{array}{c}\text { Less than } \\
100 \mathrm{~ms}\end{array}$ & $\begin{array}{l}\text { Less than } \\
3 \mathrm{~ms}\end{array}$ & Less than $16 \mathrm{~ms}$ & $\begin{array}{l}\text { Less than } \\
3 \mathrm{~ms}\end{array}$ & $\begin{array}{l}\text { Depend on } \\
\text { the class, } \\
\text { Low } \\
\text { latency } \\
\text { for class } \\
\text { C }\end{array}$ & $400 \mathrm{~ms}$ & $\begin{array}{l}\text { Less than } \\
10 \mathrm{~s}\end{array}$ & $\begin{array}{l}\text { Less than } \\
10 \mathrm{~s}\end{array}$ & - \\
\hline
\end{tabular}

proposed in order to tackle the challenges of the high data rate, the massive number of connected devices, the low latency and others. In this section, we focus on the cellular future technologies and their impacts and challenges related to the WBAN applications.

\subsection{1 $5 \mathrm{G}$ and B5G}

The fifth-generation (5G) of cellular network technology is expected to provide a very high data rate up to $10 \mathrm{Gbps}$, low latency close to the millisecond, high reliability, wide bandwidth of more than $100 \mathrm{MHz}$, high capacity, interference mitigation, massive number of connected devices and secure network [91].

In WBANs, 5G could generate significant improvements for medical applications, especially for urgent and critical applications requiring a real time transmission of data and high data rate. $5 \mathrm{G}$ can achieve a very high improvement for tele-surgery using robotic platforms with audio, video and haptic feedback [92]. It is also an efficient solution for the accurate real time continuous monitoring of patients, and for detecting the position of the patient in case of abnormal situations and it can be used for speedy transmission of medical images and videos [93]. However, power consumption of $5 \mathrm{G}$ remains an important issue in WBAN, 

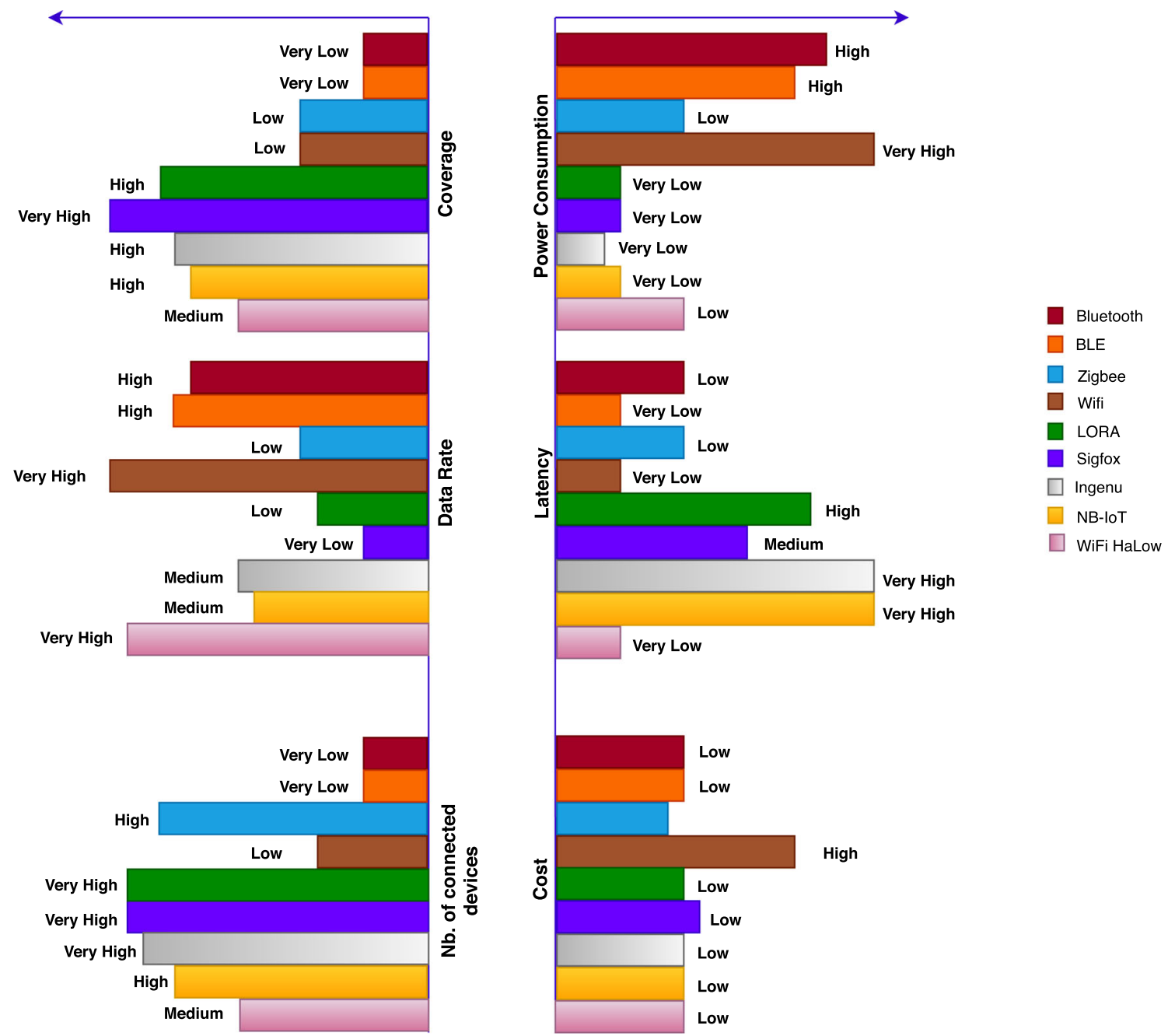

Fig. 6 Merits and demerits of Bluetooth, BLE, ZigBee, WiFi, SigFox, LoRa, Ingenu, NB-IoT and WiFi HaLow

especially for miniaturized sensors that need to operate for multiple years [94]. For beyond fifth generation (B5G) mobile communications, it should achieve a system of capacity more than 100 times compared to 5G networks, high data rate in the range of terabits per second, low latency, high reliability which can be very promising for healthcare applications in WBAN [95].

\subsection{2 $6 \mathrm{G}$}

6G will use the terahertz signal for transmission which increases the bandwidth and the data rate. The requirements of $6 \mathrm{G}$ communication technology for future healthcare are high data rate (more than 1 Tbps), low latency (less than $1 \mathrm{~ms}$ ), high reliability, high mobility support (more than $1000 \mathrm{~km} / \mathrm{h}$ ) and high bandwidth (three times higher than 5G) [96].

$5 \mathrm{G}$ and $\mathrm{B} 5 \mathrm{G}$ provides $2 \mathrm{D}$ communication, whereas $6 \mathrm{G}$ will shift to the $3 \mathrm{D}$ services using holographic communication which will revolutionize the intelligent healthcare systems especially for tele-surgery which requires a very high data rate. Other applications such as remote patient diagnostic and medical treatment supervising will be also feasible, where doctors can diagnose remotely using holographic communication which can relieve the economical and physical burden for the patients [97].

\section{WBAN medical applications and requirements}

WBAN supports many applications and has great practicality and advantages in daily life where it provides the advantages of freedom of movement, security, low power consumption, real-time data delivery and low cost. In addition to the main WBANs application in the healthcare field, to monitor patients efficiently, WBAN applications 
can be also used in entertainment, military, public security , sports and other fields [19]. The classification of WBAN applications is as follows: non-medical wearable applications and medical applications which can be wearable or implanted. In military and public safety, WBAN applications are used to improve the soldiers' performance in military situations. In this case, it is important to monitor the soldiers' vital signs and stress levels [98]. Moreover, in sports monitoring applications, the athletes physiological data collected during a game such as temperature, heart rate and body position may avoid critical situations [99]. As well patients who have recovered from an accident, can remotely perform exercises with the supervision of the medical staff [100]. For medical systems, [101] resumes the main sensors used for WBAN systems in order to monitor the development of various diseases such that diabetes, asthma, Parkinson and others. The biosensor gyroscope and the glucometer are used for diabetes monitoring. For cardiovascular disease we can use pulse oximeter, heart rate sensor and ECG sensor. Allergic sensors are used for asthma. For Parkinson disease we use motion sensors and Accelerometer. These sensors need to send their data using wireless technologies to an external medical server where it can be analyzed and stored. This can be applied based on 2, 3 or 4 tiers (see Fig. 2) depending on the architecture of the system. In order to develop a reliable and energy-saving system architecture, WBAN-based applications must overcome many difficulties, such as the battery capacity of the sensor, cost, security and others. Many research have conducted in this regards, hereafter we present many works concerning some WBAN medical applications for treatment and monitoring, where we focus on the methodology of each system, on the used technologies and the used sensors. We present some systems for diabetes patients, body temperature monitoring, asthma monitoring, Parkinson's disease, depression monitoring, Electrocardiogram ECG, oxygen saturation monitoring and finally, we present a case study about Covid-19.

\subsection{Diabetes patients}

The proposed WBAN in [76] consists of 3 tiers, for the first one, the LifeScan's OneTouch Ultra glucometer (invasive sensor) or the Combo glucometer (non-invasive sensor) are used to measure the glucose blood level, then the collected data is transferred using ZigBee or Bluetooth to a smartphone which acts as a gateway to transmit data to the AP using the IEEE 802.11, the WiFi or the GPRS communication medium. In [77], doctors can monitor remotely the patient's glucose level using an implemented glucometer sensor. ZigBee is used to send the data wirelessly from the sensor to the application system. The proposed WBAN in
[6] consists of 3 tiers. The home of a monitored patient is covered using a ZigBee network, the data is collected and processed then transmitted to the cloud service.

\subsection{Body temperature monitoring}

The sensor NTC Thermistor in [102] forward data to the PD wirelessly through ZigBee, The PD collects, processes and uploads the data through GPRS. The proposed WBAN in [103] consists of 3 tiers, where one Raspberry Pi device connect many patients and uses Bluetooth to continuously receives the messages from the sensor, then the Raspberry Pi transmit the data through cables to the main server. In [104], the sensor DS18B20 is used to monitor the temperature. The authors use an Arduino microcontroller to build a healthcare portable monitoring system. The system is equipped with an SD card, a screen, and a $2.4 \mathrm{GHz} \mathrm{WiFi}$ module to transmit data to the server. The LM35 temperature sensor in [105] send the collected data to a microcontroller via ZigBee wireless technology then the system sends its real-time data through wireless local area network to a medical monitoring database. Data collected from sensors in [106] are sent to a LoRa gateway through a LoRaWAN network, the gateway forwards the data via 3G/ $4 \mathrm{G}$ links to a server.

\subsection{Heart rate monitoring}

The system in [107] is designed using ZigBee wireless technology. The data is collected by an IR sensor the processed using an Arduino micro-controller which uses XBee network to transmit the data to a remote computer. The data collected by the sensors in [108] is processed by a microcontroller, then sent via WiFi to a main location. For efficient monitoring, a message is sent to the medical specialists in case where the collected data sent to the microcontroller is abnormal. The sensor Polar belt in [109] transmit the data to a PD through ZigBee or Bluetooth. The PD provides the user with graphical and audio interface and uses WiFi or mobile networks to transfer health data to the medical server. In [110] the patient is monitored by the heartbeat sensor TCRT1000 which is interfaced with Arduino mega microcontroller. For an effective monitoring, a message is sent via GSM to the doctor in case where the measured value exceeds the normal range.

\subsection{Asthma monitoring}

The system in [111] consists of 3 layers as follows: the sensor layer, the personal network layer and and global network layer. The location of the user is monitored by the "Qstarz BT-Q1000P" GPS, the Physical activity by a triaxial motion sensor and the Air pollution is monitored by 
the airborne Aerocet 531 Particle Counter. The base station is formed form a Nokia-N810 tablet, which is connected to the motion sensor wirelessly via IEEE 802.15.4, to the GPS receiver via Bluetooth, and wired to an airborne particle monitor via a serial USB cable. In [112], Bluetooth is used to send data from the sensor to the smartphone, the transmission of data is only done when a wheeze is detected. In [113], the collected data is transmitted from a digital bracelet and from an acoustic sensor through a LoRaWAN network. The digital bracelet is used to detect the presence of nitrite while the acoustic sensor is used to detect the wheezing sound from the chest of the patient.

\subsection{Parkinson's disease}

In [114], data transfer from the actigraphs sensors (versatile and affordable sensors) and the hub is performed using IEEE-802.15.6 standard. The system in [115] uses a number of miniature wearable wireless sensors (based on SHIMMER platform) to monitor patients with severe movement fluctuations. Gateways such as smartphones are used to send data over the Internet. The system of [116] consists from a wearable smartwatch to measure the movement of a patient and to send the data to a database server through a handheld (Smartphone). The handheld communicates via Bluetooth or $\mathrm{WiFi}$ with the smartwatch while the communication between the handheld and the server is done through HTTP requests.

\subsection{Depression monitoring}

An application was designed in [117] using an Android platform, the application is installed on a smartphone to notifies the caregiver in case of any abnormal situation by generating an alarm. The sensors used are the accelerometers and the barometric pressure sensors.

\subsection{Electrocardiogram ECG}

The system in [118] uses ZigBee wireless technology for signal transferring. The system consists of two mobile platforms, the first for signal acquisition while the second for monitoring based on the analysis of the ECG signal. In [119], a WBAN ECG remote monitoring system is presented where a power management strategy in proposed to improve system lifetime. An algorithm is also proposed in the study to classify ECG signals as stable or not. The paper [120] presents an approach for WBANs based ECG monitoring systems to identify the critical and non-critical signals using machine learning and to minimize the missclassification of critical data.

\subsection{Oxygen saturation}

In [121], Near InfraRed Spectroscopy (NIRS) is used to measure the blood oxygen saturation, then the data is sent to the doctor form the PD through GPRS/WiFi/ZigBee networks. In [122], a finger pulse oximeter is used to read the $\mathrm{SpO} 2$. The signal processing unit receive the readings via Bluetooth to take a decision to send an alarming SMS or not. The system in [123] consists of several sensors including the "My signals SPO2 sensor" oxygen saturation sensor, where data is sent to a PD by using a LoRaWAN network.

Data rate requirements of the sensors for medical systems varies from less than $1 \mathrm{kbps}$ to more than hundreds of kbps, this refers to the nature of the data to be sent, for example the heartbeat should be reported continuously whereas the temperature should be sent once in a while. For diabetes patient the data rate required is a few of kbps, for body temperature we require a very low data rate (less than $1 \mathrm{kbps}$ ), a hundred of kbps are required for the monitoring and treatment of asthma and Parkinson's patients and for the monitoring of the heart rate. Also, the Electrocardiogram ECG requires a hundred of kbps while the data rate required for the oxygen saturation monitoring is a few kbps. The power consumption highly depends on the data rate and on the used wireless technology where the choice of the wireless technology for each tier is one of the mostly important factors in WBAN systems which depends on the requirement of each system is terms of the needs of data rate, needs of coverage, the needed level of security and others. For the cost, most of the medical applications mentioned above are of low cost where this is one of the most important characteristics of WBANs. On the other hand, we have noticed the use of the smartphone in the majority of research, especially those of 3 tiers. Smartphones can collect analyze, and display the data via mobile applications. The use of smartphones reduces the cost of healthcare systems where many applications (such as cameras and accelerometers) are free [21]. However, smartphones are not effective for visually impaired patients or elderly people who cannot use the technology.

\subsection{Covid-19: a case study}

In this section, we investigate the WBAN application for Covid-19 case study. Monitoring Covid-19 remotely may highly reduce the healthcare cost and increases the hospitals' capacity by decreasing the physical presence of the patients. The needed data to collect, the required technology and the system's basic requirements will be discussed in this subsection. To monitor the symptoms, biomedical parameters like temperature, heartbeat, respiration rate and 
oxygen saturation must be collected, analysed and sent to the medical staff $[124,125]$. Such WBAN systems face a major challenge in balancing between acquisition of high fidelity data and minimizing network energy consumption and data latency while achieving a fast real time interaction in urgent case. By using biomedical sensors for health monitoring with the wireless technologies, it is possible to send medical reports to the medical staff where doctor's advice is obtained without the need of any physical movement. This WBAN system comes as a rescuer for Covid patients who need to be under monitoring, especially for elderly patients and for patients suffering from chronic diseases. Hence, two scenarios are proposed for transmitting the patient's data to the medical server. In a first scenario, for the first tier it is possible to connect the sensors to the PD using BLE or ZigBee providing a data transmission for short distance which is convenient for the WBAN's first tier. After been processed, the data must be sent to a gateway using WiFi or cellular networks. Finally, the data is received by the medical staff where the patient is diagnosed and an advice should be given in case of unstable condition. In another scenario, data is sent directly from the sensors to a gateway through one of the LPWAN technologies providing a long range, low data rate and high network lifetime which are convenient for our case. Latency remains an issue to concern, where the reception of the physiological information by the medical server at the required time is a condition for the success of the remote medical monitoring system. It is worth noting that data can be compromised by an attacker. Hence, security protocols are required in the system to protect the sensitive medical data and to maintain the patients' privacy.

\section{Sensors}

In the previous section, we have listed some WBAN systems for medical applications including the used sensors in each system. Choosing the sensor is an important part in WBANs in terms of its role, its power consumption need, its size and its lifetime. A large range of commercially sensors is used in order to measure the physiological parameters for WBAN systems. The aim of this section is to present some of many sensors found on the market for glucose monitoring, temperature monitoring, asthma monitoring, monitoring of heart rate, accelerometers and gyroscopes, and monitoring of blood pressure. Table 3 represent the position, the work technique and a classification of the data rate requirement for each one of the sensors.

The accelerometers/gyroscopes are used for monitoring of physical movement. Glucometers are used to measure the amount of glucose in the blood. Recently, this can be done through the infrared technology and the optical sensing. Temperature sensors are used to measure the temperature of the body and some of them can send signals in case of an abnormal situations. Heart rate sensors measure the amount of beats per minute. They are used to monitor the heart rate of a patient in order to diagnose heart diseases. Blood pressure sensors are used to diagnose and monitor high blood pressure which is a common risk factor for heart attacks and strokes. The important features to consider in choosing the WBAN sensors are power consumption, the size, the weight, the cost and the accuracy. It is important to achieve balance between these features because some of them are conflicting. For example, the more power a system demands, the largest the volume of its battery must be, while the need in WBAN medical systems is for a low power system with a battery of a small volume.

\section{Challenges and open issues}

The major requirements for WBANs are to achieve a secure and private network with high degree of reliability. Additional requirements such as low latency, high power efficiency and less absorption of radiations by the human body are also of high importance. This section details the most pressing challenges and some research directions to overcome them.

\subsection{Power consumption}

Power consumption still one of the major issues where long lifetime of sensors is required, especially for implanted sensors that may need to operate for several years with a disability to replace their batteries. Many research was prompted by this fact to study the ways of energy savings by maximizing the sensors' sleep time and by avoiding collision and thus avoiding the retransmission of data. Several researchers have studied techniques for recharging the battery based on body movement. Other researchers have designed a wireless self-powered sensor which is powered by the human body [152], while in [153] researchers have studied the short-range wireless transmission of energy to electronic devices.

Also in [154], a research work proposes the schedulebased charging algorithm which uses wireless power transmission technology to charge the implanted sensors in WBAN. Further, with the transition to $5 \mathrm{G}$, the optimization of power efficiency is still being investigated. Thus, more research on 5G based WBAN are still needed to achieve high power efficiency required by these networks. Here, Link adaptation and duty cycle may play an essential role [94, 155]. 
Table 3 Some market sensors

\begin{tabular}{|c|c|c|c|c|c|c|}
\hline $\begin{array}{l}\text { Field of } \\
\text { application/ } \\
\text { Disease }\end{array}$ & $\begin{array}{l}\text { Data } \\
\text { rate }\end{array}$ & Ref. & Sensor & Year & Position & Technique \\
\hline \multirow[t]{7}{*}{$\begin{array}{l}\text { Glucose } \\
\text { monitoring }\end{array}$} & Medium & {$[126,127]$} & $\begin{array}{l}\text { Guardian Real- } \\
\text { Time }\end{array}$ & 2006 & In body & $\begin{array}{l}\text { The lifetime of the sensor is } 7 \text { days. The sensor results are } \\
\text { reported with a 5-min interval and data is stored for } \\
\text { retrospective analysis. }\end{array}$ \\
\hline & Medium & {$[126,127]$} & $\begin{array}{l}\text { MiniMed 530G } \\
\text { with Enlite }\end{array}$ & 2007 & In body & $\begin{array}{l}\text { The sensor lifetime is } 7 \text { days. Glucose is reported at an interval } \\
\text { of } 5 \text {-min. The glucose data are saved for retrospective } \\
\text { analysis. }\end{array}$ \\
\hline & Medium & {$[126,127]$} & FreeStyle & 2008 & In body & $\begin{array}{l}5 \text { days is the time of insertion of the sensor, but glucose } \\
\text { concentration is not reported during the first } 10 \mathrm{~h} \text {. The } \\
\text { glucose concentration is updated every one minute and } \\
\text { stored every } 10 \mathrm{~min} \text { for retrospective analysis. }\end{array}$ \\
\hline & Medium & [128] & $\begin{array}{l}\text { Eyeglasses } \\
\text { biosensor }\end{array}$ & 2017 & Wearable & $\begin{array}{l}\text { Real-time monitoring of lactate and potassium perspiration } \\
\text { levels is provided using sensors integrated into the } \\
\text { eyeglasses' nose bridge pads. The data is sent to a remote } \\
\text { mobile wirelessly to be analyzed and visualized. }\end{array}$ \\
\hline & Medium & [129] & SwEatch & 2016 & Wearable & $\begin{array}{l}\text { The sensor transmit real-time data using Bluetooth technology } \\
\text { to a base station to be stored and visualized. The sensor can } \\
\text { continuously functions for up to three hours. }\end{array}$ \\
\hline & Medium & [130] & & 2016 & Wearable & $\begin{array}{l}\text { The sensor is integrated into a wristband that supports } \\
\text { Bluetooth technology. The sensor can operate continuously } \\
\text { up to two hours. }\end{array}$ \\
\hline & $\begin{array}{c}\text { Very } \\
\text { low }\end{array}$ & [131] & $\begin{array}{l}\text { Smart-contact } \\
\text { lens }\end{array}$ & 2014 & On body & $\begin{array}{l}\text { It is a smart-contact lens which is developed by Google[X] lab. } \\
\text { The user's is warned using a small LED when the blood } \\
\text { glucose is under or below the normal range. }\end{array}$ \\
\hline \multirow[t]{6}{*}{$\begin{array}{r}\text { Temperature } \\
\text { monitoring }\end{array}$} & $\begin{array}{c}\text { Very } \\
\text { low }\end{array}$ & {$[132,133]$} & IFever & 2018 & Wearable & $\begin{array}{l}\text { It is a Watch-type thermometer which transmit data via } \\
\text { Bluetooth to a smartphone. the sensor can operate } \\
\text { continuously up to } 648 \mathrm{~h} \text { using a cell of } 3 \mathrm{~V} \text {. Its length is } 14 \text { - } \\
19 \mathrm{~cm} \text { and its accuracy is of } \pm 0.1^{\circ} \mathrm{C} \text {. }\end{array}$ \\
\hline & $\begin{array}{l}\text { Very } \\
\text { low }\end{array}$ & {$[132,134]$} & ISense & 2018 & Wearable & $\begin{array}{l}\text { Watch-type thermometer with a rechargeable battery allowing } \\
\text { a continuous operation of } 40 \mathrm{~h} \text {. The accuracy is of } \pm 0.1^{\circ} \mathrm{C} \text {. }\end{array}$ \\
\hline & $\begin{array}{c}\text { Very } \\
\text { low }\end{array}$ & {$[132]$} & Ran's Night & 2018 & Wearable & $\begin{array}{l}\text { The sensor has a size of } 17 \times 41 \times 84 \mathrm{~mm} \text {, a weight of } 59 \mathrm{~g} \text { and } \\
\text { an accuracy of } \pm 0.5^{\circ} \mathrm{C} \text {. It measures the temperature at } 10 \\
\text { min intervals. }\end{array}$ \\
\hline & $\begin{array}{l}\text { Very } \\
\text { low }\end{array}$ & {$[132,135]$} & $\begin{array}{l}\text { FeverFrida } \\
\text { thermometer }\end{array}$ & 2018 & On body & $\begin{array}{l}\text { Disposable electrical patches that alert the user in case of a } \\
\text { high fever. It transmit data at } 4 \mathrm{~s} \text { intervals and allows up to } \\
48 \mathrm{~h} \text { continuous monitoring. }\end{array}$ \\
\hline & $\begin{array}{l}\text { Very } \\
\text { low }\end{array}$ & {$[132,134]$} & STEMP sensor & 2018 & On body & $\begin{array}{l}\text { Rechargeable patches with a size of } 33 \mathrm{~mm} \times 20 \mathrm{~mm} \times 6 \mathrm{~mm} \\
\text { and an accuracy of } \pm 0.2{ }^{\circ} \mathrm{C} \text {. }\end{array}$ \\
\hline & $\begin{array}{c}\text { Very } \\
\text { low }\end{array}$ & [136] & $\begin{array}{l}\text { Aranet medical } \\
\text { thermometer }\end{array}$ & 2020 & Wearable & $\begin{array}{l}\text { The sensor is connected to a wireless transmitter by a cable. } \\
\text { The sensor can send its measurements once every minute and } \\
\text { has an accuracy of } \pm 0.1^{\circ} \mathrm{C} \text {. }\end{array}$ \\
\hline \multirow[t]{3}{*}{$\begin{array}{l}\text { Asthma } \\
\text { monitoring }\end{array}$} & High & [137] & Propeller & 2016 & Wearable & $\begin{array}{l}\text { Propeller sensors attach to the inhalers of the patients. The } \\
\text { sensors automatically track where, when and how often the } \\
\text { patient use its medication, and they send that information to } \\
\text { an application on the phone. }\end{array}$ \\
\hline & High & [138] & AirSonea & 2013 & Wearable & $\begin{array}{l}\text { Asthma monitor synchronize with smartphones to enable a } \\
\text { patient at any time to easily monitor wheezing symptoms. } \\
\text { The device is placed on the neck and it is allowed to record } \\
\text { sounds made by breathing. Data is transmitted via Bluetooth } \\
\text { to a smartphone to be analyzed and recorded. }\end{array}$ \\
\hline & High & [139] & Wing & 2015 & Wearable & $\begin{array}{l}\text { The sensor measure the maximum speed and volume a patient } \\
\text { can exhale during one second. A smartphone is used to } \\
\text { display the results. }\end{array}$ \\
\hline
\end{tabular}


Table 3 (continued)

\begin{tabular}{|c|c|c|c|c|c|c|}
\hline $\begin{array}{l}\text { Field of } \\
\text { application/ } \\
\text { Disease }\end{array}$ & $\begin{array}{l}\text { Data } \\
\text { rate }\end{array}$ & Ref. & Sensor & Year & Position & Technique \\
\hline \multirow[t]{6}{*}{$\begin{array}{l}\text { Heartrate } \\
\text { monitoring }\end{array}$} & High & {$[140]$} & BH1790GLC & 2019 & Wearable & $\begin{array}{l}\text { Heartbeat sensor designed to give outputs about heartbeat } \\
\text { when the user place its finger on it. This output can be } \\
\text { connected directly in order measure the heartbeats rate bi a } \\
\text { microcontroller. }\end{array}$ \\
\hline & High & {$[141]$} & TCRT 1000 & & Wearable & $\begin{array}{l}\text { Embedded Included in a wrist wearable device where the } \\
\text { output is sent to computer for processing via Bluetooth. }\end{array}$ \\
\hline & High & [141] & $\begin{array}{l}\text { Smart QHeart } \\
\text { Rate Sensor }\end{array}$ & & Wearable & $\begin{array}{l}\text { It consists of an IR led embedded in a clip fixed to the earlobe } \\
\text { or to the finger and a detector part attached on the body. }\end{array}$ \\
\hline & High & [142] & BH1790GLC & 2017 & Wearable & $\begin{array}{l}\text { Low power sensor of size } 2.8 \times 2.8 \times 1 \mathrm{~mm} \text {. It is used to monitor } \\
\text { the heart rate.It integrates a LED driver and a green light } \\
\text { detection photodiode. }\end{array}$ \\
\hline & High & {$[143]$} & Polar H10 & 2017 & Wearable & $\begin{array}{l}\text { Very accurate sensor, functions for around } 400 \text { hours. The } \\
\text { sensor uses Bluetooth to transmit the data wirelessly to a } \\
\text { smartphone. }\end{array}$ \\
\hline & High & [144] & Pulse sensor & 2011 & Wearable & $\begin{array}{l}\text { It is a plug and play heart rate sensor for Arduino. Has a } \\
\text { diameter of } 16 \mathrm{~mm} \text { and a thickness of } 3 \mathrm{~mm} \text { and a power } \\
\text { consumption of } 20 \mathrm{~mW} \text {. Has a } 24 \text { cable which is terminated } \\
\text { with a standard male header. }\end{array}$ \\
\hline \multirow[t]{4}{*}{$\begin{array}{l}\text { Accelerometers } \\
\text { and gyroscope }\end{array}$} & High & {$[145]$} & $\begin{array}{l}\text { MPU-9250 } \\
\text { Invensense }\end{array}$ & 2016 & Wearable & $\begin{array}{l}\text { A 9-axis Motion Tracking device, Gyroscope/Accelerometer/ } \\
\text { Magnetometer Sensor with a size of } 3 \times 3 \times 1 \mathrm{~mm} \text { and a power } \\
\text { consumption of } 9.3 \text { microAmper. }\end{array}$ \\
\hline & High & [146] & $\begin{array}{l}\text { MMA 8451Q, } \\
\text { digital } \\
\text { accelerometer }\end{array}$ & 2017 & Wearable & $\begin{array}{l}\text { The sensor MMA8451Q is a three-axis accelerometer, it is } \\
\text { low-power, with } 14 \text { bits of resolution and has a size of } \\
3 \times 3 \times 1 \mathrm{~mm} \text {. }\end{array}$ \\
\hline & High & [147] & LSM 303 DLHC & 2011 & wearable & $\begin{array}{l}\text { The sensor LSM303DLHC combines a } 3 \text {-axis accelerometer } \\
\text { with a 3-axis magnetometer. Has a size of } 3 \times 5 \times 1 \mathrm{~mm} \text {. The } \\
\text { board operates from } 2.5 \text { to } 5.5 \mathrm{~V} \text {. }\end{array}$ \\
\hline & & {$[148]$} & BNO055 & 2016 & Wearable & $\begin{array}{l}\text { The sensor BNO055 combines a 3-axis accelerometer, a 3-axis } \\
\text { gyroscope, a } 3 \text {-axis geomagnetic and a microcontroller. Its } \\
\text { size is } 3.8 \times 5.2 \times 1.1 \mathrm{~mm} \text {. }\end{array}$ \\
\hline \multirow[t]{3}{*}{$\begin{array}{l}\text { Blood pressure } \\
\text { monitoring }\end{array}$} & Low & [149] & $\begin{array}{l}\text { Blood pressure } \\
\text { reader, } \\
\text { biosignals plux }\end{array}$ & 2018 & Wearable & $\begin{array}{l}\text { Sensor of size } 72 \times 70 \times 32 \mathrm{~mm} \text { with an accuracy of } \pm \\
3 \mathrm{mmHg} \text { and a weight of } 140 \mathrm{~g} \text { including the adapter. }\end{array}$ \\
\hline & Low & {$[150]$} & $\begin{array}{l}\text { IProven BPM- } \\
\text { 337BT }\end{array}$ & 2017 & Wearable & $\begin{array}{l}\text { IProven is a Wrist Blood Pressure Monitor Watch of size } \\
80 \times 65 \times 22 \mathrm{~mm} \text { and a weight of } 120 \mathrm{~g} \text {. }\end{array}$ \\
\hline & Low & [151] & $\begin{array}{l}\text { Omron M7 } \\
\text { Intelli IT }\end{array}$ & 2015 & Wearable & $\begin{array}{l}\text { The M7 Intelli IT of size } 124 \times 90 \times 161 \mathrm{~mm} \text { is a blood } \\
\text { pressure monitor using Bluetooth with a weight of } 390 \mathrm{~g} \text { and } \\
\text { an accuracy of } \pm 3 \mathrm{mmHg} \text {. }\end{array}$ \\
\hline
\end{tabular}

\subsection{Absorption of radiations by the human body}

The absorption of radio-frequency radiation may have negative impact on the human body, specially for sensitive organs. For instance, the eyes exposure to an infrared radiation is a high concern where the exposure to radiations of rate of absorption per unit weight of $8 \mathrm{~W} / \mathrm{Kg}$ in the head or chest for a time of 15 minutes can leads to the risk of tissue damage [156] knowing that the maximum permitted exposure rate value, is $2 \mathrm{~W} / \mathrm{kg}$ in Europe and $1.6 \mathrm{~W} / \mathrm{kg}$ in the USA. So, one of the principal challenges in WBAN is to reduce the radiations rate [157]. To minimize the energy absorbed by the human body, the sensors must maintain their power efficiency to maintain a low-power absorption [158]. Thus, WBAN equipment should be able to run less time and use low power transmission. This is related to the choice of technology and the methodology of the system where LPWAN technologies can become one of the solutions due to their low transmission power and low duty cycles. 


\subsection{Security and privacy issues}

As mentioned in section 2.2.3, several security prototypes for WBANs have been described in the literature. Despite these studies, security and privacy of medical data in WBANs remains two huge challenges requiring appropriate mechanisms where currently, much research is going, and several open issues exist such as:

First, in the near future, WBAN will become more pervasive, where the patient data will be shared with family members, doctors, hospitals, pharmacies and others which requires a high level of consistent policy sets to protect the patient's privacy.

Second, WBANs may be vulnerable to various attacks, the authors of [159] classified these security attacks into two kind of attacks: external and misbehaviour. For the external attack, it can be easily handled by traditional security mechanisms while it cannot handle the misbehaviour attacks which require more research studies.

Third, another area that needs attention is the area of trust management. Trust is the degree to which a node can be considered trustworthy, reliable and safe when interacting with other nodes. A survey about latest research about trust management for WBAN is presented in [160].

Fourth, complex security mechanisms for WBANs require more power resources, so a balance between many factors such as security, power consumption, efficiency and practicality is very crucial and need more researches to achieve the widespread use of the medical applications in WBANs.

Some recent analysis about security and privacy issues are presented hereinafter. In [161], the paper details the security and privacy issues and challenges raised by the increasing use of the IoT. Then, the authors analyze existing solutions that address these challenges, and they suggest new solutions to address the existing ones. Also in [162], the study provides an IoT security analysis, where it examines the potential threats and identifies the countermeasures and their limitations paying an attention to the mechanisms of security of the IoT protocol. The work of [163] outlines and discusses the general attacks that can plague the IoT devices and networks. It then evaluates LoRaWAN's security mechanisms, and whether that security can be effectively compromised. The paper [164] studies the possible threats within pacemakers. The paper attempts to show if it is possible to hack a current model of an industrial pacemaker.

\subsection{LPWAN technologies protocols}

LPWAN are promising technologies due to their low power consumption and their long range, but it remains some modification to do in the LPWAN protocols in order to support e-health applications. Urgent and On-demand transmissions are highly requested in the medical applications, but are not supported in many LPWAN protocols, such as LoRaWAN, where the protocol is mainly designed for Uplink transmissions. Other challenges related to the mobility of the patients have to be addressed by these protocols since many of them do not support the mobile things communication [165].

\subsection{WBAN performance: reliability and quality of service}

Transmission reliability is a critical issue ensuring the performance of a WBAN [101] where the monitored data must be received correctly by the medical staff or it can be fatal if a life-threatening event is not detected. Reliability includes the delivery of data in a reasonable time, and a guaranteed delivery of data. The unreliability has main causes as: low transmission range, interference, inefficient routing and others [166].

Quality of service (QoS) is one of the basic characteristics to achieve a reliable network. Reliable QoS indicates the arrive of the packets on correct order and on time [19]. According to [167], QoS is a key part of the medical information transmission in WBAN, where critical applications require the highest QoS. High QoS requires a high throughput which is an important part to improve the system performance. The throughput is proportional to the packet payload and inversely proportional to the transmission delay. QoS also requires a minimum delay in order to ensure the data effectiveness. Another requirement is to maximize the network lifetime by controlling the main sources of energy lost [48]. 5G may be a good candidate in helping WBAN system to achieve high QoS since this generation will ensure high data rate, high reliability and very low latency. However, this may increase the energy consumption. Therefore, we have to compromise between these factors to achieve a good performance.

\subsection{Interference}

Interference in WBANs is one of the major open issues which essentially needs to be addressed. The requirement to support many different sensors on the human body in WBAN, in addition to the coexistence of many WBANs within the same area, makes the coordination between the WBAN systems necessary and challenging [168]. The problem of interference can also arise between WBANs and the other wireless networks in the ISM bands leading to a low health data reliability, energy inefficiency and high latency caused by the signal distortion. 
Recently, several methods have been proposed in the literature to mitigate interference in WBANs. Hereinafter, some of these published studies. In [169], a scheme based upon Efficient Mitigating Rules "EMR" is proposed at the level of the central coordinator to mitigate interference. The work of [170] formulates the channel selection of the coexisting coordinators in WBAN. Two algorithms are proposed to achieve the Nash Equilibrium of the formulated game. Paper [171] considers node-level interference mitigation instead of treating each WBAN as a whole. The main objective of [172] is the scheduling of the nodes in the WBAN in an interference free manner. Authors proposes a priority-based scheduling algorithm where the nodes priority is taken into consideration for scheduling the body sensors. The work of [173] proposes a scheduling technique to mitigate interference under a machine-tomachine based medical WBAN. In the work of [174], a distributed TDMA scheme is proposed to minimize the probability of collision between nodes in coexisting WBANs. Furthermore, it is well-known that power control is used to mitigate interference, where many studies prove its efficiency in reducing interference. In order to mitigate the inter-WBANs interference, [175] proposes a power control game-based approach which is based on the patients' social interaction information. In [176], an adaptive transmission power control MAC protocol is proposed in order to improve the network throughput using a single channel and transceiver. By controlling the power of transmission, concurrent transmissions are enabled without interference between them. In [177], a new power-controlled MAC is proposed to increase the probability of concurrent transmissions and the durability of battery life. The survey [178] detailed the power control algorithms and classified them according to their approaches.

\section{Conclusion and future scope}

With the huge developments in wireless technologies and with the arrival of $5 \mathrm{G}$ technology, the growth of Wireless Body Area Network (WBAN) has accelerated in order to improve the human life quality. This paper aims at highlighting the most recent advances in WBAN systems as well as future challenges. The main features of WBAN systems such as power consumption, interference, latency, security, privacy, reliability and the choice of a suitable sensor, are well detailed in this paper. We also discussed the state of the art of several wireless communication technologies to determine their suitability for WBANs medical applications, including the newly emerged LPWAN communication technologies. The role to play by $5 \mathrm{G}, \mathrm{B} 5 \mathrm{G}$, and $6 \mathrm{G}$ and their impact on the healthcare systems are also presented. Further, we point out a list of methodologies and features of a wide range of WBAN medical applications and we surveyed a wide range of sensors used in these systems regarding their size, position and their operating technique. This paper highlighted also a set of challenges and future research axes related to security and privacy of the WBAN data, adapting the emerging technologies protocols to the e-health applications, the health impact of the technologies, interference and others.

For the future perspectives of WBAN, they can be inspired from the Covid-19 pandemic. The actual situation of Covid-19 raises up several challenges related to the ehealthcare system. This pandemic proves the efficiency of WBANs in decreasing the medical cost and the contact between the patient and the medical specialists, increasing the hospitals' capacity, and tracking the suspected cases of Covid-19. Efficient and scalable e-health systems become crucial for the future of the health care. However, the huge increase in the e-health devices raises several issues such as the network capacity and the interference management. Also, high speed data rate may be one of the main requirements for the future WBAN systems in order to carrying massive data (such as video and image data) fastly to the medical centre. With the coming of $5 \mathrm{G}$ and beyond, high data rate could be ensured where this technology is expected to increase the data rate $100 \times$ the speed of $4 \mathrm{G}$. Finally, with the high spread of the WBAN systems, the data security and the patient privacy raise also high challenges. Then, efficient security techniques should be developed to protect the medical data due to its sensitivity and criticality. Furthermore, ethical laws should be considered and developed to keep the patient privacy safe.

\section{References}

1. World Health Organization (WHO). Geneva.

2. Mathers, C. D., \& Loncar, D. (2005). Updated projections of global mortality and burden of disease, 2002-2030: data sources, methods and results. Geneva: World Health Organization.

3. Pramanik, P. K. D., Nayyar, A., \& Pareek, G. (2019). Wban: Driving e-healthcare beyond telemedicine to remote health monitoring: Architecture and protocols. In Telemedicine technologies (pp. 89-119). Elsevier.

4. Gardašević, G., Katzis, K., Bajić, D., \& Berbakov, L. (2020). Emerging wireless sensor networks and internet of things technologies-foundations of smart healthcare. Sensors, 20(13), 3619.

5. Nelson, B. D., Karipott, S. S., Wang, Y., \& Ong, K. G. (2020). Wireless technologies for implantable devices. Sensors, 20(16), 4604.

6. Wang, J., Zhang, Z., Zheng, Y., Zuo, L., \& Kim, J.-U. (2013). A multitiers service architecture based diabetes monitoring for elderly care in hospital. International Journal of Multimedia and Ubiquitous Engineering, 8(3), 387-398. 
7. Rubani, Q., Gupta, S. H., \& Kumar, A. (2019). Design and analysis of circular patch antenna for wban at terahertz frequency. Optik, 185, 529-536.

8. Chatterjee, S., Chatterjee, S., Choudhury, S., Basak, S., Dey, S., Sain, S., Ghosal, K. S., Dalmia, N. \& Sircar, S. (2017). Internet of things and body area network-an integrated future. In 2017 IEEE 8th Annual Ubiquitous Computing, Electronics and Mobile Communication Conference (UEMCON) (pp. 396-400). IEEE.

9. Crosby, G. V., Ghosh, T., Murimi, R., \& Chin, C. A. (2012). Wireless body area networks for healthcare: A survey. International Journal of Ad Hoc, Sensor \& Ubiquitous Computing, $3(3), 1$.

10. Nguyen, C. T., Saputra, Y. M., Van Huynh, N., Nguyen, N. -T., Khoa, T. V., Tuan, B. M., Nguyen, D. N., Hoang, D. T., Vu, T. X., Dutkiewicz, E. et al. (2020). Enabling and emerging technologies for social distancing: A comprehensive survey. arXiv preprint arXiv:2005.02816.

11. Guo, C., Tian, P., \& Choo, K.-K.R. (2020). Enabling privacyassured fog-based data aggregation in e-healthcare systems. IEEE Transactions on Industrial Informatics, 17, 1948-1957.

12. Pal, A. (2020). Sensor based computational model to control the spreading of covid-19 virus in a smart city. Studies in Indian Place Names, 40(70), 4983-4994.

13. https://ec.europa.eu/eurostat/statistics explained/index.php. eurostat statistics explained, healthcare expenditure statistics, 20220.

14. Keehan, S. P., Cuckler, G. A., Poisal, J. A., Sisko, A. M., Smith, S. D., Madison, A. J., Rennie, K. E., Fiore, J. A., \& Hardesty, J. C. (2020). National health expenditure projections, 2019-28: Expected rebound in prices drives rising spending growth: National health expenditure projections for the period 2019-2028. Health Affairs, 39(4), 704-714.

15. ERICSSON. Define the future of the industrial iot, 4 February 2020.

16. Hayajneh, T., Almashaqbeh, G., Ullah, S., \& Vasilakos, A. V. (2014). A survey of wireless technologies coexistence in wban: analysis and open research issues. Wireless Networks, 20(8), 2165-2199.

17. Khan, R. A., \& Pathan, A.-S.K. (2018). The state-of-the-art wireless body area sensor networks: A survey. International Journal of Distributed Sensor Networks, 14(4), 1550147718768994.

18. Hasan, K., Biswas, K., Ahmed, K., Nafi, N. S., \& Islam, M. S. (2019). A comprehensive review of wireless body area network. Journal of Network and Computer Applications, 143, 178-198.

19. Salayma, M., Al-Dubai, A., Romdhani, I., \& Nasser, Y. (2017). Wireless body area network (wban) a survey on reliability, fault tolerance, and technologies coexistence. ACM Computing Surveys (CSUR), 50(1), 1-38.

20. Chen, M., Gonzalez, S., Vasilakos, A., Cao, H., \& Leung, V. C. M. (2011). Body area networks: A survey. Mobile networks and applications, 16(2), 171-193.

21. Ghamari, M., Janko, B., Sherratt, R. S., Harwin, W., Piechockic, R., \& Soltanpur, C. (2016). A survey on wireless body area networks for ehealthcare systems in residential environments. Sensors, 16(6), 831.

22. Olatinwo, D. D., Abu-Mahfouz, A., \& Hancke, G. (2019). A survey on lpwan technologies in wban for remote health-care monitoring. Sensors, 19(23), 5268.

23. Cavallari, R., Martelli, F., Rosini, R., Buratti, C., \& Verdone, R. (2014). A survey on wireless body area networks: Technologies and design challenges. IEEE Communications Surveys \& Tutorials, 16(3), 1635-1657.
24. Cao, H., Leung, V., Chow, C., \& Chan, H. (2009). Enabling technologies for wireless body area networks: A survey and outlook. IEEE Communications Magazine, 47(12), 84-93.

25. Qadri, Y. A., Nauman, A., Zikria, Y. B., Vasilakos, A. V., \& Kim, S. W. (2020). The future of healthcare internet of things: A survey of emerging technologies. IEEE Communications Surveys \& Tutorials, 22(2), 1121-1167.

26. Narwal, B., \& Mohapatra, A. K. (2020). A survey on security and authentication in wireless body area networks. Journal of Systems Architecture, 113, 101883.

27. Pandey, I., Dutta, H. S., \& Banerjee, J. S. (2019). Wban: a smart approach to next generation e-healthcare system. In 2019 3rd International Conference on Computing Methodologies and Communication (ICCMC) (pp. 344-349). IEEE.

28. Poongodi, T., Rathee, A., Indrakumari, R., \& Suresh, P. (2020). Iot sensing capabilities: sensor deployment and node discovery, wearable sensors, wireless body area network (wban), data acquisition. In Principles of Internet of Things (IoT) Ecosystem: Insight Paradigm (pp. 127-151). Springer.

29. Antonescu, B., \& Basagni, S. (2013). Wireless body area networks: challenges, trends and emerging technologies. In Proceedings of the 8th international conference on body area networks (pp. 1-7).

30. Al-Janabi, S., Al-Shourbaji, I., Shojafar, M., \& Shamshirband, S. (2017). Survey of main challenges (security and privacy) in wireless body area networks for healthcare applications. Egyptian Informatics Journal, 18(2), 113-122.

31. Seferagić, A., Famaey, J., De Poorter, E., \& Hoebeke, J. (2020). Survey on wireless technology trade-offs for the industrial internet of things. Sensors, 20(2), 488.

32. Kumar, P., \& Lee, H.-J. (2012). Security issues in healthcare applications using wireless medical sensor networks: A survey. sensors, 12(1), 55-91.

33. Mainanwal, V., Gupta, M., \& Upadhayay, S. K. (2015). A survey on wireless body area network: Security technology and its design methodology issue. In 2015 international conference on innovations in information, embedded and communication systems (ICIIECS) (pp. 1-5). IEEE.

34. Sawaneh, I. A., Sankoh, I., \& Koroma, D. K. (2017). A survey on security issues and wearable sensors in wireless body area network for healthcare system. In 2017 14th International Computer Conference on Wavelet Active Media Technology and Information Processing (ICCWAMTIP) (pp. 304-308). IEEE.

35. Di Pietro, R., Mancini, L. V., \& Mei, A. (2006). Energy efficient node-to-node authentication and communication confidentiality in wireless sensor networks. Wireless Networks, 12(6), 709-721.

36. Li, M., Yu, S., Lou, W., \& Ren, K. (2010). Group device pairing based secure sensor association and key management for body area networks. In 2010 Proceedings IEEE INFOCOM (pp. 1-9). IEEE.

37. Venkatasubramanian, K. K., Banerjee, A., \& Gupta, S. K. S. (2009). Pska: Usable and secure key agreement scheme for body area networks. IEEE Transactions on Information Technology in Biomedicine, 14(1), 60-68.

38. Liu, X., Zhang, R., \& Zhao, M. (2019). A robust authentication scheme with dynamic password for wireless body area networks. Computer Networks, 161, 220-234.

39. Almuhaideb, A. M., \& Alqudaihi, K. S. (2020). A lightweight and secure anonymity preserving protocol for wban. IEEE Access, 8, 178183-178194.

40. Konan, M., \& Wang, W. (2019). A secure mutual batch authentication scheme for patient data privacy preserving in wban. Sensors, 19(7), 1608.

41. Khan, F. H., Shams, R., Rizvi, H. H., Qazi, F., et al. (2018). A secure crypto base authentication and communication suite in 
wireless body area network (wban) for iot applications. Wireless Personal Communications, 103(4), 2877-2890.

42. Gupta, A., Tripathi, M., \& Sharma, A. (2020). A provably secure and efficient anonymous mutual authentication and key agreement protocol for wearable devices in wban. Computer Communications, 160, 311-325.

43. Ramli, S. N., Ahmad, R., Abdollah, M. F., \& Dutkiewicz, E. (2013). A biometric-based security for data authentication in wireless body area network (wban). In 2013 15th international conference on advanced communications technology (ICACT) (pp. 998-1001). IEEE.

44. Negra, R., Jemili, I., \& Belghith, A. (2016). Wireless body area networks: Applications and technologies. Procedia Computer Science, 83, 1274-1281.

45. Kakria, P., Tripathi, N. K., \& Kitipawang, P. (2015). A real-time health monitoring system for remote cardiac patients using smartphone and wearable sensors. International Journal of Telemedicine and Applications, 2015, 8. https://doi.org/10.1155/ 2015/373474.

46. Chen, H., Wanqing, W., \& Lee, J. (2010). A wban-based realtime electroencephalogram monitoring system: design and implementation. Journal of medical systems, 34(3), 303-311.

47. Hadjem, M., Salem, O., \& Naït-Abdesselam, F. (2014). An ecg monitoring system for prediction of cardiac anomalies using wban. In 2014 IEEE 16th International Conference on e-Health Networking, Applications and Services (Healthcom) (pp. 441-446). IEEE.

48. Chakraborty, C., Gupta, B., \& Ghosh, S. K. (2013). A review on telemedicine-based wban framework for patient monitoring. Telemedicine and e-Health, 19(8), 619-626.

49. Ivanov, S., Foley, C., Balasubramaniam, S., \& Botvich, D. (2012). Virtual groups for patient wban monitoring in medical environments. IEEE Transactions on Biomedical Engineering, 59(11), 3238-3246.

50. Le, T. T. T., \& Moh, S. (2015). Interference mitigation schemes for wireless body area sensor networks: A comparative survey. Sensors, 15(6), 13805-13838.

51. Meharouech, A., Elias, J., \& Mehaoua, A. (2019). Moving towards body-to-body sensor networks for ubiquitous applications: A survey. Journal of Sensor and Actuator Networks, 8(2), 27.

52. Misra, S., Moulik, S., \& Chao, H.-C. (2015). A cooperative bargaining solution for priority-based data-rate tuning in a wireless body area network. IEEE Transactions on Wireless Communications, 14(5), 2769-2777.

53. de Silva, B., Natarajan, A., \& Motani, M. (2009). Inter-user interference in body sensor networks: Preliminary investigation and an infrastructure-based solution. In 2009 Sixth International Workshop on Wearable and Implantable Body Sensor Networks (pp. 35-40). IEEE.

54. Domenicali, D. \& Di Benedetto, M. -G. (2007). Performance analysis for a body area network composed of ieee 802.15. 4a devices. In 2007 4th Workshop on Positioning, Navigation and Communication (pp. 273-276). IEEE.

55. Kim, S., Kim, S., Kim, J.-W., \& Eom, D.-S. (2012). A beacon interval shifting scheme for interference mitigation in body area networks. Sensors, 12(8), 10930-10946.

56. Shah, A. M., Abdelmaboud, A., Mahmood, K., ul Hassan, M., \& Saeed, M. K. (2018). ehealth wban: Energy-efficient and priority-based enhanced ieee802. $15.6 \mathrm{csma} / \mathrm{ca}$ mac protocol. International Journal of Advanced Computer Science and Applications, 9(4), 82-87.

57. Riazul Islam, S. M., Avazov, N., Dobre, O. A., \& Kwak, K.-S. (2016). Power-domain non-orthogonal multiple access (noma) in $5 \mathrm{~g}$ systems: Potentials and challenges. IEEE Communications Surveys \& Tutorials, 19(2), 721-742.
58. Ma, Z., \& Bao, J. (2019). Sparse code multiple access (scma). In Multiple Access Techniques for $5 G$ Wireless Networks and Beyond (pp. 369-416). Springer.

59. Wang, J., Sun, Y., \& Ji, Y. (2018). Priority-based capacity and power allocation in co-located wbans using stackelberg and bargaining games. The Journal of Supercomputing, 74(7), 3114-3147.

60. Yuan, X., Li, C., Ye, Q., Zhang, K., Cheng, N., Zhang, N., \& Shen, X. (2018). Performance analysis of ieee 802.15. 6-based coexisting mobile wbans with prioritized traffic and dynamic interference. IEEE Transactions on Wireless Communications, $17(8), 5637-5652$.

61. Guo, Z., Harris, I. G., Tsaur, L. -F., \& Chen, X. (2015). An ondemand scatternet formation and multi-hop routing protocol for ble-based wireless sensor networks. In 2015 IEEE wireless communications and networking conference (WCNC) (pp. 1590-1595). IEEE.

62. Siekkinen, M., Hiienkari, M., Nurminen, J. K. \& Nieminen, J. (2012). How low energy is bluetooth low energy? comparative measurements with zigbee/802.15. 4. In 2012 IEEE wireless communications and networking conference workshops (WCNCW) (pp. 232-237). IEEE.

63. Minakshi, J. A.-H. (2016). An overview of wireless body area network (wban) using zigbee technology. International Journal of Scientific Development and Research (IJSDR), 1, 2455-2631.

64. Chung, Y.-F., \& Liu, C.-H. (2013). Design of a wireless sensor network platform for tele-homecare. Sensors, 13(12), 17156-17175.

65. Cecilia, H., de Schatz, V., Medeiros, H. P., Schneider, F. K., \& Abatti, P. J. (2012). Wireless medical sensor networks: Design requirements and enabling technologies. Telemedicine and e-Health, 18(5), 394-399.

66. Arefin, M. T., Ali, M. H., \& Haque, A. K. M. F. (2017). Wireless body area network: An overview and various applications. Journal of Computer and Communications, 5(7), 53-64.

67. Arunapriya, K., \& Kumar, P. (2018). Lifi for medical care using visible light communication. International Journal of Applied Engineering Research ISSN, 13(11), 0973-4562.

68. Porselvi, S., Bhagyalakshmi, L., \& Sanjay, K. S. (2017). Healthcare monitoring systems using li-fi networks. Innovare Journal of Engineering \& Technology, 5(2), 1-4.

69. Sharma, R. R., Sanganal, A., et al. (2014). Li-fi technology: Transmission of data through light. International Journal of Computer Technology and Applications, 5(1), 150.

70. Mekki, K., Bajic, E., Chaxel, F., \& Meyer, F. (2019). A comparative study of lpwan technologies for large-scale iot deployment. ICT Express, 5(1), 1-7.

71. Sundaram, J. P. S., Wan, D., \& Zhao, Z. (2019). A survey on lora networking: Research problems, current solutions and open issues. IEEE Communications Surveys \& Tutorials, 22, 371-388.

72. www.semtech.com. Lora modulation basics, semtech corporation, May 2015.

73. Augustin, A., Yi, J., Clausen, T., \& Townsley, W. M. (2016). A study of lora: Long range \& low power networks for the internet of things. Sensors, 16(9), 1466.

74. https://lora-alliance.org/resource-hub/lorawantm-specification v11. Lora alliance, lorawan specification v1.1, 2017.

75. Antolín, D., Medrano, N., Calvo, B., \& Pérez, F. (2017). A wearable wireless sensor network for indoor smart environment monitoring in safety applications. Sensors, 17(2), 365.

76. Huzooree, G., Khedo, K. K., \& Joonas, N. (2016). Wireless body area network system architecture for real-time diabetes monitoring. In International Conference on Emerging Trends in Electrical, Electronic and Communications Engineering (pp. 262-271). Springer. 
77. Al Rasyid, M. U. H., Saputra, F. A., \& Christian, A. (2016). Implementation of blood glucose levels monitoring system based on wireless body area network. In 2016 IEEE International Conference on Consumer Electronics-Taiwan (ICCE-TW) (pp. 1-2). IEEE.

78. Queralta, J. P., Gia, T. N., Zou, Z., Tenhunen, H., \& Westerlund, T. (2019). Comparative study of lpwan technologies on unlicensed bands for $\mathrm{m} 2 \mathrm{~m}$ communication in the iot: Beyond lora and lorawan. Procedia Computer Science, 155, 343-350.

79. Baker, S. B., Xiang, W., \& Atkinson, I. (2017). Internet of things for smart healthcare: Technologies, challenges, and opportunities. IEEE Access, 5, 26521-26544.

80. Da Silva, W. R., Oliveira, L., Kumar, N., Rabêlo, R. A. L., Marins, C. N. M., \& Rodrigues, J. J. P. C. (2018). An internet of things tracking system approach based on lora protocol. In 2018 IEEE Global Communications Conference (GLOBECOM) (pp. $1-7)$. IEEE.

81. Song, Y., Lin, J., Tang, M., \& Dong, S. (2017). An internet of energy things based on wireless lpwan. Engineering, 3(4), 460-466.

82. Mekki, K., Bajic, E., Chaxel, F., \& Meyer, F. (2018). Overview of cellular lpwan technologies for iot deployment: Sigfox, lorawan, and nb-iot. In 2018 ieee international conference on pervasive computing and communications workshops (percom workshops) (pp. 197-202). IEEE.

83. Mikhaylov, K., Stusek, M., Masek, P., Petrov, V., Petajajarvi, J., Andreev, S., Pokorny, J., Hosek, J., Pouttu, A., \& Koucheryavy. Y. (2018). Multi-rat lpwan in smart cities: Trial of lorawan and nb-iot integration. In 2018 IEEE International Conference on Communications (ICC), pages 1-6. IEEE.

84. Li, Y., Cheng, X., Cao, Y., Wang, D., \& Yang, L. (2017). Smart choice for the smart grid: Narrowband internet of things (nb-iot). IEEE Internet of Things Journal, 5(3), 1505-1515.

85. Raza, U., Kulkarni, P., \& Sooriyabandara, M. (2017). Low power wide area networks: An overview. IEEE Communications Surveys \& Tutorials, 19(2), 855-873.

86. Knyazev, N. S., Chechetkin, V. A., \& Letavin, D. A. (2017). Comparative analysis of standards for low-power wide-area network. In 2017 Systems of Signal Synchronization, Generating and Processing in Telecommunications (SINKHROINFO) (pp. 1-4). IEEE.

87. Bankov, D., Khorov, E., Lyakhov, A., \& Famaey, J. (2020). Resource allocation for machine-type communication of energyharvesting devices in wi-fi halow networks. Sensors, 20(9), 2449.

88. http://www.wi-fi.org/discover-wifi/wi-fi halow. Wi-fi halow.

89. Ojha, T., Misra, S., \& Raghuwanshi, N. S. (2015). Wireless sensor networks for agriculture: The state-of-the-art in practice and future challenges. Computers and Electronics in Agriculture, 118, 66-84.

90. Finnegan, J., \& Brown, S. (2018). A comparative survey of lpwa networking. arXiv preprint arXiv:1802.04222.

91. Sodhro, A. H. \& Shah, M. A. (2017). Role of $5 \mathrm{~g}$ in medical health. In 2017 International Conference on Innovations in Electrical Engineering and Computational Technologies (ICIEECT) (pp. 1-5). IEEE.

92. Soldani, D., Fadini, F., Rasanen, H., Duran, J., Niemela, T., Chandramouli, D., Hoglund, T., Doppler, K., Himanen, T., Laiho, J. et al. (2017). 5g mobile systems for healthcare. In 2017 IEEE 85th Vehicular Technology Conference (VTC Spring) (pp. 1-5). IEEE.

93. Selem, E., Fatehy, M., \& El-Kader, S. M. A. (2019). E-health applications over $5 \mathrm{~g}$ networks: challenges and state of the art. In 2019 6th International Conference on Advanced Control Circuits and Systems (ACCS) \& 2019 5th International Conference on New Paradigms in Electronics \& information Technology (PEIT) (pp. 111-118). IEEE.

94. Jones, R. W. \& Katzis, K. (2018). 5g and wireless body area networks. In 2018 IEEE Wireless Communications and Networking Conference Workshops (WCNCW) (pp. 373-378). IEEE.

95. Ms Huq, K., Busari, S. A., Rodriguez, J., Frascolla, V., Bazzi, W., \& Sicker, D. C. (2019). Terahertz-enabled wireless system for beyond-5g ultra-fast networks: A brief survey. IEEE Network, 33(4), 89-95.

96. Chowdhury, M. Z., Shahjalal, M., Ahmed, S., \& Jang, Y. M. (2019). 6g wireless communication systems: Applications, requirements, technologies, challenges, and research directions. arXiv preprint arXiv:1909.11315.

97. Nayak, S., \& Patgiri, R. (2020). 6 g communication technology: A vision on intelligent healthcare. arXiv preprint arXiv:2005. 07532.

98. Jovanov, E., Lords, A. D., Raskovic, D., Cox, P. G., Adhami, R., \& Andrasik, F. (2003). Stress monitoring using a distributed wireless intelligent sensor system. IEEE Engineering in Medicine and Biology Magazine, 22(3), 49-55.

99. Yang, G.-Z., \& Yang, G. (2006). Body sensor networks (Vol. 1). Berlin: Springer.

100. Tseng, Y. -C., Wu, C. -H., Wu, F. -J., Huang, C. -F., King, C. T., Lin, C. -Y., Sheu, J. -P., Chen, C. -Y., Lo, C. -Y., Yang, C. W. et al. (2009). A wireless human motion capturing system for home rehabilitation. In 2009 Tenth International Conference on Mobile Data Management: Systems, Services and Middleware (pp. 359-360). IEEE.

101. Barakah, D. M. \& Ammad-uddin, M. (2012). A survey of challenges and applications of wireless body area network (wban) and role of a virtual doctor server in existing architecture. In 2012 Third International Conference on Intelligent Systems Modelling and Simulation (pp. 214-219). IEEE.

102. Meena, R., Ravishankar, S., \& Gayathri, J. (2014). Monitoring physical activities using wban. International Journal of Computer Science and Information Technologies, 5(4), 5880-5886.

103. Hassan, H. J., Hadi, N. K., \& Taqi, A. K. (2018). Implementation of wireless body area network based patient monitoring system. Journal of Information Engineering and Applications, $8(4)$.

104. Purnomo, D. D., \& Basari, (2016). Design of pulse rate and body temperature monitoring system with arduino via wifi and android-based gadget. International Journal of Technology and Engineering Studies, 2(5), 140-148.

105. Mansor, H., Shukor, M. H. A., Meskam, S. S., Rusli, N. Q. A. M., \& Zamery, N. S. (2013). Body temperature measurement for remote health monitoring system. In 2013 IEEE International conference on smart instrumentation, measurement and applications (ICSIMA) (pp. 1-5)

106. Mdhaffar, A., Chaari, T., Larbi, K., Jmaiel, M., \& Freisleben, B. (2017). Iot-based health monitoring via lorawan. In IEEE EUROCON 2017-17th International Conference on Smart Technologies (pp. 519-524). IEEE.

107. Venugopal, K. \& Kumar, A. (2013). Centralized heart rate monitoring and automated message alert system using wban.

108. Otto, C. A., Jovanov, E., \& Milenkovic, A. (2006). A wbanbased system for health monitoring at home. In 2006 3rd IEEE/ EMBS International Summer School on Medical Devices and Biosensors (pp. 20-23). IEEE.

109. Devi, J. G., Prabha, S. P. R. J., Ramalakshmi, S. P., \& Keerthika, R. (2016). Wireless patient aid alert system using microcontroller. International Journal of Advanced Research Trends in Engineering and Technology, 3(14).

110. Kioumars, A. H. \& Tang, L. (2011). Wireless network for health monitoring: heart rate and temperature sensor. In 2011 Fifth 
International Conference on Sensing Technology (pp. 362-369). IEEE.

111. Keränen, N., Särestöniemi, M., Partala, J., Hämäläinen, M., Reponen, J., Seppänen, T., Iinatti, J., \& Jämsä, T. (2013). Ieee802. 15.6-based multi-accelerometer wban system for monitoring parkinson's disease. In 2013 35th Annual International Conference of the IEEE Engineering in Medicine and Biology Society (EMBC) (pp. 1656-1659). IEEE.

112. Patel, S., Chen, B. -R., Buckley, T., Rednic, R., McClure, D., Tarsy, D., Shih, L., Dy, J., Welsh, M., \& Bonato, P. (2010). Home monitoring of patients with parkinson's disease via wearable technology and a web-based application. In 2010 annual international conference of the IEEE engineering in medicine and biology (pp. 4411-4414). IEEE.

113. Ray, R., Mallick, D. K., \& Dash, S. R. (2019). Intelligent energy-efficient healthcare models integrated with iot and lora network. In Sensors for Health Monitoring (pp. 157-174). Elsevier.

114. Contreras, R., Huerta, M., Sagbay, G., LLumiguano, C., Bravo, M., Bermeo, A., Clotet, R., \& Soto, A. (2016). Tremors quantification in parkinson patients using smartwatches. In 2016 IEEE Ecuador Technical Chapters Meeting (ETCM), (pp. 1-6). IEEE.

115. Saha, J., Biswas, S., Bhattacharyya, T., \& Chowdhury, C. (2016). A framework for monitoring of depression patient using wban. In 2016 International Conference on Wireless Communications, Signal Processing and Networking (WiSPNET) (pp. 410-415). IEEE.

116. Lee, H. -J., Kim, D. -O., Kang, B. -J., \& Ban, S. -W. (2011). Mobile embedded health-care system working on wireless sensor network. In 2011 Third International Conference on Communications and Mobile Computing (pp. 161-164). IEEE.

117. Yu, F., \& Liu, J. (2015). System design for wearable blood oxygen saturation and pulse measurement device. Procedia Manufacturing, 3, 1187-1194.

118. Alshorman, S., Jaber, F. T. \& Bensaali, F. (2015). A wireless oxygen saturation and heart rate monitoring and alarming system based on the qatar early warning scoring system. In 2015 International Conference on Computational Science and Computational Intelligence (CSCI) (pp. 787-790). IEEE.

119. Sarma, J., \& Biswas, R. (2020). Vlsi based adaptive power management architecture for ecg monitoring in wban. In 2020 33rd International Conference on VLSI Design and 2020 19th International Conference on Embedded Systems (VLSID) (pp. 113-118). IEEE.

120. Roy, A. P., Chatterjee, S., Maji, P., \& Mondal, H. K. (200) Classification of ecg signals for iot-based smart healthcare applications using wban. In 2020 International Symposium on Devices, Circuits and Systems (ISDCS) (pp. 1-4). IEEE.

121. Oletic, D., Arsenali, B., \& Bilas, V. (2011). Towards continuous wheeze detection body sensor node as a core of asthma monitoring system. In International Conference on Wireless Mobile Communication and Healthcare (pp. 165-172). Springer.

122. Seto, E. Y. W., Giani, A., Shia, V., Wang, C., Yan, P., Yang, A. Y., Jerrett, M., \& Bajcsy, R. (2009). A wireless body sensor network for the prevention and management of asthma. In 2009 IEEE International Symposium on Industrial Embedded Systems (pp. 120-123). IEEE.

123. Islam, M. S., Islam, M. T., Almutairi, A. F., Beng, G. K., Misran, N., \& Amin, N. (2019). Monitoring of the human body signal through the internet of things (iot) based lora wireless network system. Applied Sciences, 9(9), 1884.

124. El-Rashidy, N., El-Sappagh, S., Islam, S. M., El-Bakry, H. M., \& Abdelrazek, S. (2020). End-to-end deep learning framework for coronavirus (covid-19) detection and monitoring. Electronics, 9(9), 1439.
125. Jia, L., Li, K., Jiang, Y., Guo, X. et al. (2020). Prediction and analysis of coronavirus disease 2019. arXiv preprint arXiv:2003. 05447.

126. Wang, H.-C., \& Lee, A.-R. (2015). Recent developments in blood glucose sensors. Journal of Food and Drug Analysis, 23(2), 191-200.

127. McGarraugh, G. (2009). The chemistry of commercial continuous glucose monitors. Diabetes technology \& therapeutics, $11(\mathrm{~S} 1), \mathrm{S}-17$.

128. Sempionatto, J. R., Nakagawa, T., Pavinatto, A., Mensah, S. T., Imani, S., Mercier, P., \& Wang, J. (2017). Eyeglasses based wireless electrolyte and metabolite sensor platform. Lab on a Chip, 17(10), 1834-1842.

129. Glennon, T., O'Quigley, C., McCaul, M., Matzeu, G., Beirne, S., Wallace, G. G., et al. (2016). 'sweatch': A wearable platform for harvesting and analysing sweat sodium content. Electroanalysis, 28(6), 1283-1289.

130. Gao, W., Emaminejad, S., Nyein, H. Y. Y., Challa, S., Chen, K., Peck, A., Fahad, H. M., Ota, H., Shiraki, H., Kiriya, D., et al. (2016). Fully integrated wearable sensor arrays for multiplexed in situ perspiration analysis. Nature, 529(7587), 509-514.

131. Tamura, T., Huang, M., \& Togawa, T. (2018). Current developments in wearable thermometers. Advanced Biomedical Engineering, 7, 88-99.

132. Bruen, D., Delaney, C., Florea, L., \& Diamond, D. (2017). Glucose sensing for diabetes monitoring: recent developments. Sensors, 17(8), 1866.

133. http://www.vipose.com/. ifever, 2018.

134. iSense: smartphone baby temperature sensor and alarm. Stemp smart temperature patch, 2018.

135. http://feversmart.com/. Fiver smart patch thermometer, 2018.

136. https://www.aranet.com/. Aranet, 2020.

137. Propeller. https://www.propellerhealth.com/, 2016. Accessed = 2016.

138. https://www.medgadget.com/2013/09/airsonea video.html. Sparo labs for asthma device, 2013. Accessed: 092013.

139. https://engineering.wustl.edu/news/Pages/Sparo-Labs-launchescrowdfunding-campaign-for-asthmadevice.aspx. Wing, 2015.

140. https://www.pantechsolutions.net/heart-beat sensor. Heart beat sensor,pantechsolutions, 2019.

141. https://www.elprocus.com/heartbeat-sensor-working applica tion/. Smart q, heartbeat sensor-working \& application.

142. https://www.rohm.com/sensor-shield-support/heart-rate sensor. heart rate sensor module bh1790glc, 2017.

143. https://www.polar.com/blog/new-polar-h10-heart-rate-sensor 2017/. Polar h10 heart rate sensor, 2017.

144. https://pulsesensor.com/products/pulse-sensor amped. Pulse sensor, 2011.

145. https://invensense.tdk.com/wpcontent/uploads/2015/02/ PSMPU-9250A-01-v1.1.pdf. Mpu-9250 product specification, invensense, 2016.

146. https://www.nxp.com/docs/en/data sheet/MMA8451Q.pdf. Data sheet: Document number: Mma8451q, 022017.

147. https://cdn-shop.adafruit.com/datasheets/LSM303DLHC.PDF. Lsm303dlhc ultra compact high performance e-compass $3 \mathrm{~d}$ accelerometer and 3d magnetometer module, 2011.

148. https://www.boschsensortec.com/media/boschsensortec/down loads/. Data sheet: Bno055 intelligent absolute orientation sensor, 2016.

149. https://biosignalsplux.com/datasheets/BPR. Data sheet blood pressure reader sen, 2018.

150. https://medaval.ie/docs/manuals/iProven BPM-337BT-Manual. pdf. iproven bpm-337bt, pressure monitor instruction manual, 2017. 
151. https://www.ajmedical.se/media/wysiwyg/ajm/pdf. Automatic upper arm blood pressure monitor $\mathrm{m} 7$ intelli it (hem-7322t-e) instruction manual, 2015.

152. Heinzel, A., Hebling, C., Müller, M., Zedda, M., \& Müller, C. (2002). Fuel cells for low power applications. Journal of Power Sources, 105(2), 250-255.

153. Kurs, A., Karalis, A., Moffatt, R., Joannopoulos, J. D., Fisher, P., \& Soljačić, M. (2007). Wireless power transfer via strongly coupled magnetic resonances. science, 317(5834), 83-86.

154. Rabby, M. K. M., Alam, M. S., \& Shawkat, M. S. T. S. A. (2019). A priority based energy harvesting scheme for charging embedded sensor nodes in wireless body area networks. PloS One, 14(4), e0214716.

155. Liu, Y., Davaslioglu, K., \& Gitlin, R. D. (2017). Energy efficiency optimization of channel access probabilities in ieee 802.15. 6 uwb wbans. In 2017 IEEE wireless communications and networking conference (WCNC) (pp. 1-6). IEEE.

156. Ahmed, G., Ul Islam, S., Shahid, M., Akhunzada, A., Jabbar, S., Khan, M. K., Riaz, M., \& Han, K. (2018). Rigorous analysis and evaluation of specific absorption rate (sar) for mobile multimedia healthcare. IEEE Access, 6, 29602-29610.

157. Foster, K. R. (2000). Thermal and nonthermal mechanisms of interaction of radio-frequency energy with biological systems. IEEE Transactions on Plasma Science, 28(1), 15-23.

158. Fernandez, M., Espinosa, H. G., Guerra, D., Peña, I., Thiel, D. V., \& Arrinda, A. (2020). Rf energy absorption in human bodies due to wearable antennas in the $2.4 \mathrm{ghz}$ frequency band. Bioelectromagnetics, 41(1), 73-79.

159. Ng, H. S., Sim, M. L., \& Tan, C. M. (2006). Security issues of wireless sensor networks in healthcare applications. BT Technology Journal, 24(2), 138-144.

160. Ayed, S., Chaari, L., \& Fares, A. (2020). A survey on trust management for wban: Investigations and future directions. Sensors, 20(21), 6041.

161. Moganedi, S. \& Mtsweni, J. (2017). Beyond the convenience of the internet of things: Security and privacy concerns. In 2017 IST-Africa Week Conference (IST-Africa) (pp. 1-10). IEEE.

162. Grammatikis, P. I. R., Sarigiannidis, P. G., \& Moscholios, I. D. (2019). Securing the internet of things: Challenges, threats and solutions. Internet of Things, 5, 41-70.

163. Ingham, M., Marchang, J., \& Bhowmik, D. (2020). Iot security vulnerabilities and predictive signal jamming attack analysis in lorawan. IET Information Security, 14(4), 368-379.

164. Beavers, J. L., Faulks, M., \& Marchang, J. (2019). Hacking nhs pacemakers: a feasibility study. In 2019 IEEE 12th International Conference on Global Security, Safety and Sustainability (ICGS3) (pp. 206-212). IEEE

165. Ayoub, W., Samhat, A. E., Nouvel, F., Mroue, M., \& Prévotet, J.-C. (2018). Internet of mobile things: Overview of lorawan, dash7, and nb-iot in lpwans standards and supported mobility. IEEE Communications Surveys \& Tutorials, 21(2), 1561-1581.

166. Abbasi, U. F., Awang, A. \& Hamid, N. H. (2014). A cross-layer opportunistic mac/routing protocol to improve reliability in wban. In The 20th Asia-Pacific Conference on Communication (APCC2014) (pp. 36-41). IEEE.

167. Li, C., Li, H. -B. \& Kohno, R. (2009). Performance evaluation of ieee 802.15. 4 for wireless body area network (wban). In 2009 IEEE International conference on communications workshops (pp. 1-5). IEEE.

168. Alam, M. M. \& Hamida, E. B. (2015). Interference mitigation and coexistence strategies in ieee 802.15. 6 based wearable body-to-body networks. In International Conference on Cognitive Radio Oriented Wireless Networks) (pp. 665-677). Springer.

169. Shaik, M. F. \& Subashini, M. M. (2020). A new approach for interference mitigation in multiple wban using emr-rules. In
2020 6th International Conference on Advanced Computing and Communication Systems (ICACCS) (pp. 266-269). IEEE.

170. George, E. M., \& Jacob, L. (2020). Interference mitigation for coexisting wireless body area networks: Distributed learning solutions. IEEE Access, 8, 24209-24218.

171. Movassaghi, S., Abolhasan, M., Smith, D., \& Jamalipour, A. (2014). Aim: Adaptive internetwork interference mitigation amongst co-existing wireless body area networks. In 2014 IEEE global communications conference (pp. 2460-2465). IEEE.

172. Pradhan, P. P., \& Bhattacharjee, S. (2020). Interference minimized slot scheduling for coexisting wbans: Delay and prioritybased approach. In Optical and Wireless Technologies (pp. 193-206). Springer.

173. Park, R. C., Jung, H., \& Jo, S.-M. (2014). Abs scheduling technique for interference mitigation of $\mathrm{m} 2 \mathrm{~m}$ based medical wban service. Wireless Personal Communications, 79(4), 2685-2700.

174. Ali, M., Moungla, H., Younis, M. \& Mehaoua, A. (2016). Distributed scheme for interference mitigation of wbans using predictable channel hopping. In 2016 IEEE 18th international conference on e-Health networking, applications and services (Healthcom) (pp. 1-6). IEEE.

175. Zhang, Z., Wang, H., Wang, C., \& Fang, H. (2013). Interference mitigation for cyber-physical wireless body area network system using social networks. IEEE Transactions on Emerging Topics in Computing, 1(1), 121-132.

176. Li, P., Geng, X., \& Fang, Y. (2009). An adaptive power controlled mac protocol for wireless ad hoc networks. IEEE Transactions on Wireless Communications, 8(1), 226-233.

177. Marchang, J., Douglas, R., Ghita, B., Lancaster, D., \& Sanders, B. (2018). Dynamic neighbour aware power-controlled mac for multi-hop ad-hoc networks. Ad Hoc Networks, 75, 119-134.

178. Pradhan, N. L., \& Saadawi, T. (2011). Power control algorithms for mobile ad hoc networks. Journal of Advanced Research, 2(3), 199-206.

Publisher's Note Springer Nature remains neutral with regard to jurisdictional claims in published maps and institutional affiliations.

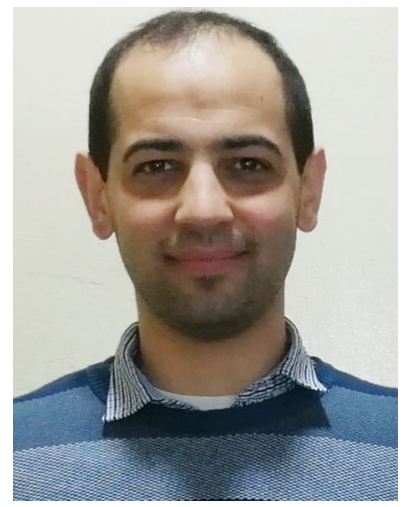

Houssein Taleb received the B.Sc. and the M.Sc. in Applied Physics from the Lebanese University in 2011 and 2013, respectively. From February 2019, he has been a Ph.D. student in Electronics at the Université de Nantes, France. His current research interests include E-Health, Internet of Things and Wireless Sensor Networks. 


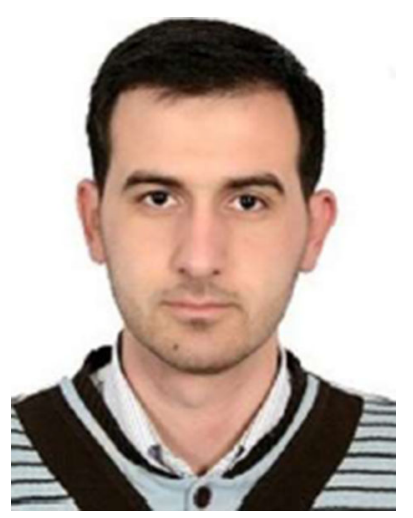

Abbass Nasser received the B.Sc. and the M.Sc. in Electronics from the Lebanese University in 2010 and 2012, respectively. From 2012 to 2016, he has been an instructor at the Department of Computer Science, American University of Culture and Education (AUCE), Lebanon. From April 2013 to December 2016, he has been a Ph.D. student at the Université de Bretagne Occidendale (UBO), France. From June 2017 to May 2019, he was post-doc researcher at Ensta-Bretagne, France. Since June 2019, he has been research fellow at Ensta-Bretagne. In October 2020, he was a visiting professor at Polytech-Nantes, France, and in September 2021 he has was a visiting researcher at Lab-STICC - UBO - France. Dr. Nasser is the supervisor and/or the co-director of several $\mathrm{PhD}$ projects. He has more than 40 research papers published in International Journals and Conferences. He serves as guest editor for Sensors journal and Topic Editor for Electronics Journal. He serves as reviewer for several IEEE, MDPI and Elsevier Journals. His current research interests include E-Health, Cognitive Radio, Intelligent Reflecting Surfaces, Internet of Things and Wireless Sensor Networks.

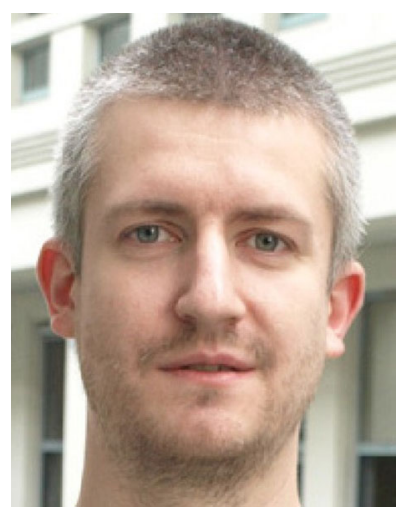

Guillaume Andrieux graduated in electrical engineering from Polytech Nantes and received the M.S. degree in Telecommunications from the University of Nantes, France, in 2000. He received the Ph.D. degree in electrical engineering and the Habilitation à Diriger des Recherches degree from the University of Nantes, France, in 2004 and 2018, respectively. He is currently an Associate Professor at the Networks and Telecommunications Department, University of Nantes. His current research interests are digital communications, antenna processing, wireless sensor networks, energy efficiency in wireless networks, and channel estimation.

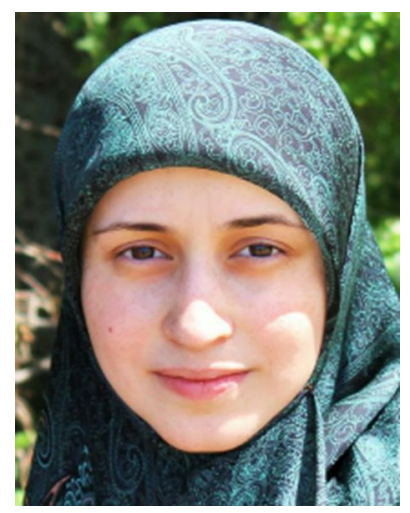

Nour Charara holds a Ph.D. in Electronics and Telecommunications from the University of Applied Sciences of Western Switzerland HES-SO, Fribourg, Switzerland \& Doctoral School of Sciences and Technology Lebanese University, Beirut, Lebanon, in 2015. Also she received Research Master Degree in Signal, Telecom, Image, and Speech (STIP) from the Lebanese University, Doctoral School of Sciences and Technology in 2010. Currently, she is Assistant Professor on the Computer Science department at the faculty of Science and Literature on the American University of Culture and Education (AUCE). Her research interests include Artificial Intelligence in image/video Analysis, IoT and e-health.

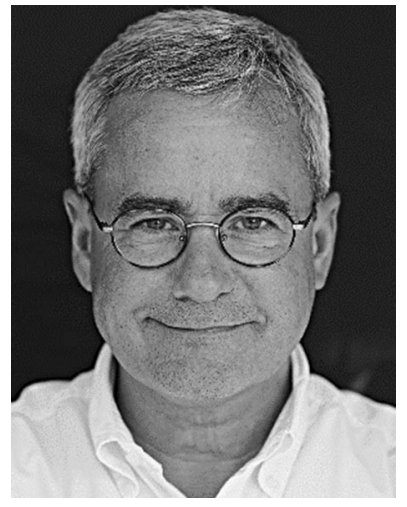

Eduardo Motta Cruz received the Ph.D. degree in Electronics and the "Habilitation à Diriger des Recherches" degree in Electronics from the University of Rennes 1, France, in 1991 and 2008 , respectively. He has more than 25 years of industrial experience in the management of $R \& D$ and engineering teams of manufacturers and operators of telecommunications networks. Since 2015 he is Full Professor at the Polytechnic School of the University of Nantes, and Director of the "Systems, Networks and Telecommunications" Engineering Department. He is member of the CNRS Laboratory IETR, "Institut d'Electronique et des Technologies du numéRique", at the BEAMS team (BEAm Antennas up to Mm and Submm waves). He is (co)inventor of 20 patents, (co)author of more than 40 journal papers and publications in international conferences and workshops, and has supervised more than 30 master students, Ph.D. students and postdoctoral Fellows. His research interests include 4G and 5G cellular Networks and Antennas, Communications Systems, IoT applied to Industry 4.0/Smart Cities/e-health, Radio Propagation and Metamaterials. 